\title{
On the Shape and Synthesis of Extended Fluorenones: Recent Advances and Upcoming Challenges
}

Benjamin Large*[a] and Damien Prim*[b]

[a] Dr. B. Large, University of York, Department of Chemistry, YO10 5DD, York, United Kingdom

E-mail: benjamin.large@york.ac.uk

[b] Pr. Dr. D. Prim, ILV, Université Paris-Saclay, UVSQ, CNRS, 78035 Versailles, France

E-mail: damien.prim@uvsq.fr

URL: https://www.ilv.uvsq.fr/catalysis-and-heterocycles

Twitter: @ILV_UMR8180

\begin{abstract}
Extended fluorenones represent a particular class of polycyclic compounds. The extension of the aromatic system through the fusion of additional benzene rings onto the central tricyclic fluorenone gives rise to an impressive topological diversity and allows to modulate the properties of the original molecule. The multiple ways this aromatic extension can be joined onto the tricyclic core generates multiple spatial arrangements from linear, angular, mixed linear-angular, to helical. This extensive range of shapes and their subsequent applications has piqued the curiosity of the scientific community, who now creatively compete to develop selective and direct routes to these valuable molecular architectures. Potential applications of these diverse domains have also driven the development of strategies for the installation of various substituents at selected sites of the carbon backbone. This review intends to provide the reader with an up-to-date overview of the methods for constructing extended fluorenones and their properties.
\end{abstract}

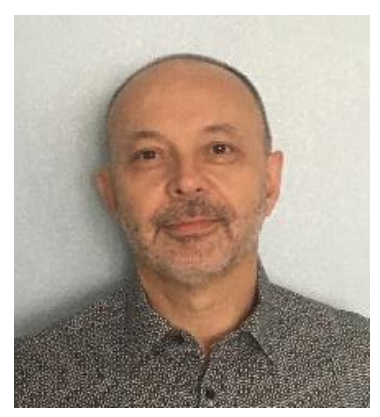

Damien Prim studied chemistry at the Paul Verlaine University of Metz, where he received his Ph.D. in organic chemistry in 1994 under the guidance of Prof. Gilbert Kirsch. After a postdoctoral period with Prof. Léon Ghosez at UC Louvain-la-Neuve, Belgium, he was appointed as an assistant professor at Paul Verlaine University of Metz. In 1999, he moved to Pierre et Marie Curie University in Paris as a CNRS research associate. He then moved to the University of Versailles St Quentin as an assistant professor in 2001. In 2005, he was appointed as a full professor of chemistry at the University of Versailles St Quentin. Since 2020, he has been head of the Graduate School of Chemistry at the University of Paris-Saclay. His research focuses on homogeneous and heterogenous catalysis and the syntheses and properties of polycyclic molecular architectures.

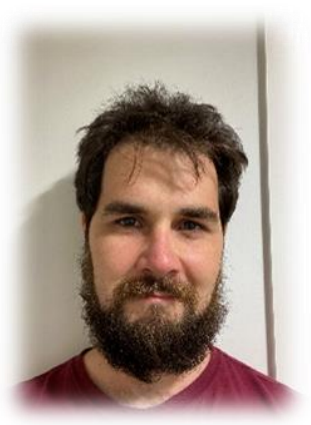

Benjamin Large studied chemistry at the Ecole Nationale Supérieure de Chimie de Montpellier, France, and then joined Damien Prim's team at the University of Versailles St Quentin as a Ph.D. student. His thesis was focused on the selective $\mathrm{C}-\mathrm{H}$ functionalization of naphthalene and its derivatives. After obtaining his Ph.D. in 2019, Benjamin joined AnneKathrin Duhme-Klair's team as a postdoctoral research associate at the University of York (UK), and is currently working on the development of artificial metalloenzymes 


\section{Introduction}

Fluorenones are important structural molecular platforms composed of a condensed planar arrangement of at least three carbocycles including a central cyclopentanone flanked by two aromatic systems. The $9 \mathrm{H}$-fluoren-9-one represents the smallest member of this family and has been known for almost 150 years since its discovery in $1873 .{ }^{1}$ The interest of the scientific community towards these molecules stems from their wide applications in material sciences, ${ }^{2,3,4,5,6}$ sensing ${ }^{7}$ and as potent biological active compounds. ${ }^{8,9,10,11}$ Such versatility of fluorenones is achieved either through the selective installation of diverse substituents or the conjugation of additional molecules onto the cyclic backbone with the aim of fine-tuning their properties; thus, creating the demand for more efficient synthetic methodologies. ${ }^{10}$

In extended fluorenones, additional benzene rings may be diversely condensed to the central fluorenone unit giving rise to multiple molecular layouts and enhanced molecular diversity (Figure 1). As shown, a "linear" extension represents the condensation of an aromatic unit to face $b$ of the $9 \mathrm{H}$ fluoren-9-one leading to benzo[ $b]$-fused fluorenone backbone. In contrast, condensation onto faces $a$ or $c$ leads to a non-linear or angular extension of the topology of fluorenone derivatives.

If one additional ring affords three possible tetracyclic molecular architectures, two additional rings give rise to seven mixed or differential orientations, combining linear and angular extensions, leading to a clear increase of molecular topologies and penta- or hexa-cyclic derivatives. 
"linear"
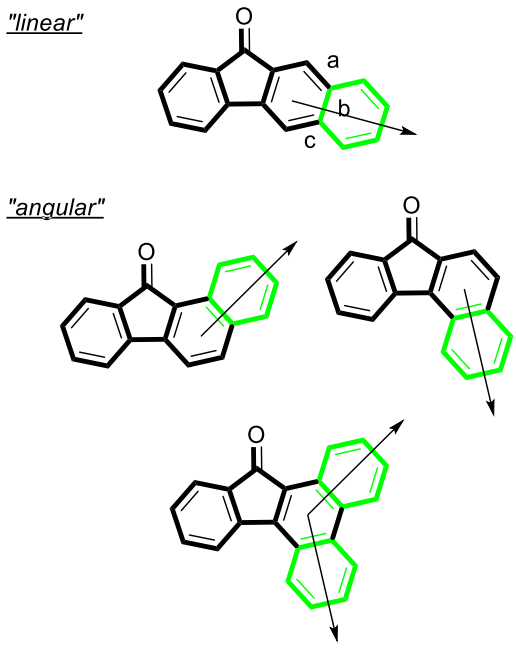

"bis linear"
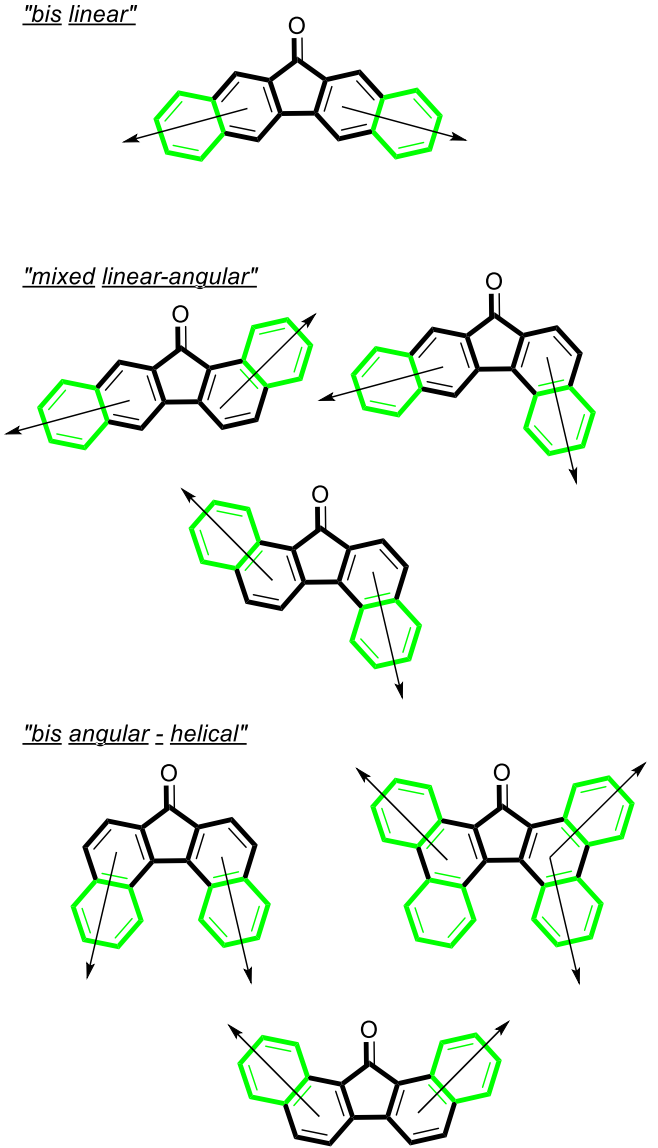

FIGURE 1 - Topological diversity of extended fluorenones

The synthesis of the $9 \mathrm{H}$-fluoren-9-one unit has been described for almost one century. ${ }^{12}$ More recently, the synthetic protocols leading to functionalized $9 \mathrm{H}$-fluoren-9-one derivatives have been reviewed, ${ }^{10}$ however, there is a notable absence of literature coverage dedicated to extended fluorenones. The presence of one or more additional rings increases the possibilities of ring sequence, but above all requires specific synthesis strategies and access to functionalized precursors. As an example, access to bis linear pentacyclic targets can be envisioned from five different pathways reflecting the specificity of extended fluorenones (Figure 2). Firstly, the joint construction of two A-C or B-C rings is typical of pentacyclic structures and is not encountered in the lower homologue series. Secondly, the 
construction of the central ring $\mathbf{A}$ is also a distinct feature requiring a naphthalene precursor. Finally, if the oxidative process and ring contraction routes are similar to what has been previously described in the fluorenone series, the synthetic pathway to the required precursor is undoubtedly characteristic of the extended structure.

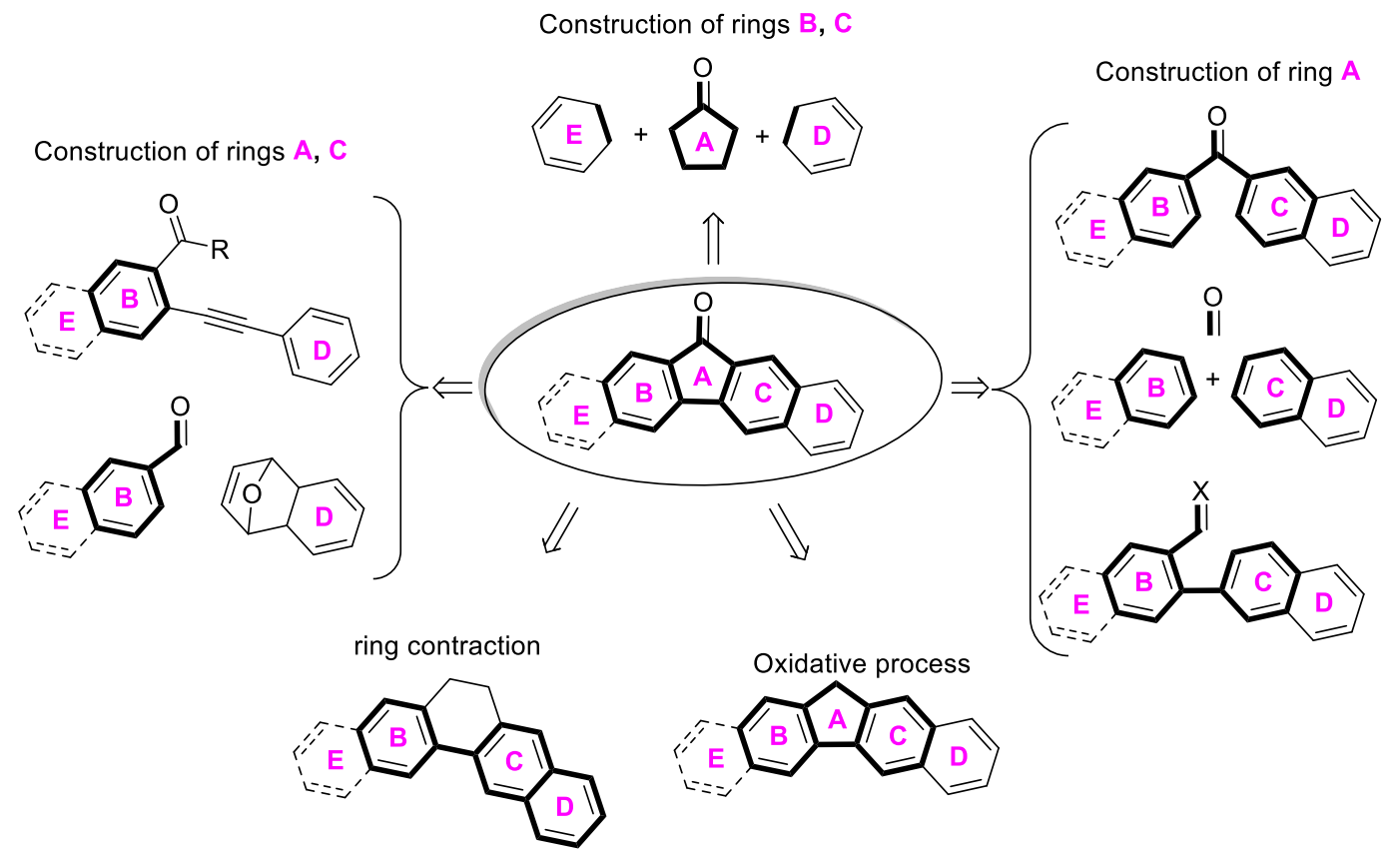

FIGURE 2 - Strategies of construction

Moving from simple fluorenones to extended fluorenone required the development of targeted strategies. This review intends to provide an overview of main strategies for accessing extended fluorenones. This review is divided in several sections depending on the way the extended fluorenone core is constructed and the number of bonds created during the critical construction step. The first two sections are devoted to the oxidation of parent fluorenone derivatives and ring contraction from a six-membered ring to the central cyclopentanone unit. Emphasis will next be placed on approaches for the construction of ring A from naphthalene precursors. In this context, naphthyl-aryl ketones, arynes intermediates and aryl-naphthalenes bearing functional groups such as carboxaldehydes, nitriles, carboxylic acids will be considered. Finally, this review will focus on strategies involving the joint construction of rings $B$ and $C$, and further rings $A$ and $C$.

\section{To extended fluorenones through oxidative processes from parent fluorene derivatives}

Oxidation of the $\mathrm{sp}^{3}$ carbon of fluorene most likely represents the earliest approach for the preparation of fluorenones. Recent literature survey shows a plethora of reagents and conditions for benzylic $\mathrm{C}-\mathrm{H}$ oxidation processes because the chemo selective oxidation of benzylic $\mathrm{sp}^{3} \mathrm{C}-\mathrm{H}$ provides can be efficiently used for the late-stage functionalization of more complex molecules. ${ }^{13}$ Moreover, the oxidation of fluorene to fluorenone is among the benchmark transformations usually considered to evaluate oxidation systems. Although apparently easy to set, this strategy is poorly exemplified in the extended fluorenone series as it requires the synthesis of extended fluorenes as a prerequisite. The 
most efficient methods that have appeared in recent literature, involve both homogeneous and heterogeneous conditions (Figure 3 ).
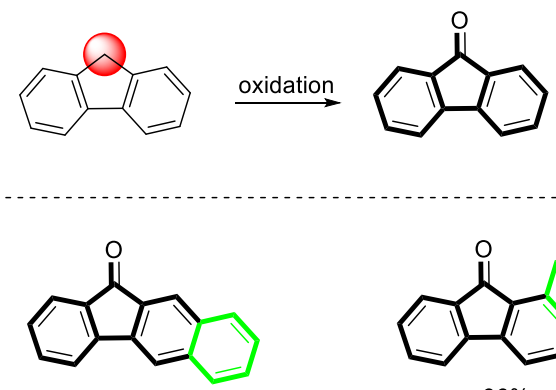

$\mathrm{O}_{2}$, Ni-MnOx, $n$-octane, $80^{\circ} \mathrm{C}$ quant. $\mathrm{KOH}$-graphene, air, DLF, rt $95 \%$

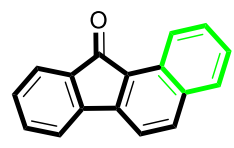

$96 \%$

$\mathrm{KOH}$-graphene air, DMF, rt

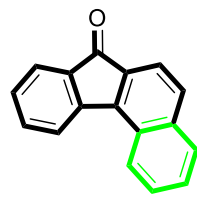

$\mathrm{KOH}$-graphene, air, DMF, rt, 95\% $\mathrm{NaBiO}_{3}, \mathrm{AcOH} /$ Acetone $/ \mathrm{H}_{2} \mathrm{O}$, reflux, $54 \%$
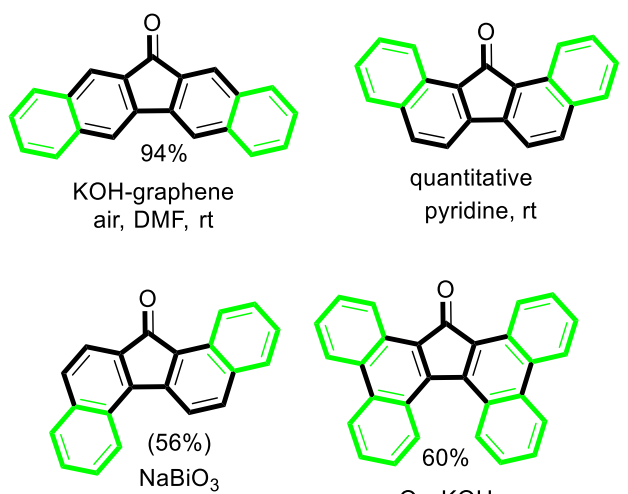

$\mathrm{AcOH} /$ Acetone $/ \mathrm{H}_{2} \mathrm{O}$

reflux, $4 \mathrm{~h}-48 \mathrm{~h}$

$\mathrm{O}_{2}, \mathrm{KOH}$

18-crown-6

DME, $100^{\circ} \mathrm{C}$

FIGURE 3 - Oxidative processes

Heterogeneous $\mathrm{Ni}-\mathrm{MnOx}$ nanoparticles have been proved efficient to promote oxidation in quantitative yields of a broad substrate scope including benzo[b]fluorene. ${ }^{14}$

Similarly, aerobic oxidation using graphene-supported $\mathrm{KOH}$ in DMF at room temperature (Scheme 1) appears to be a highly efficient route to a broad range of extended fluorenes and well adapted to linear and angular tetra- and pentacyclic targets. ${ }^{15} \mathrm{~A}$ plausible mechanism considers the enhanced acidic character of the benzylic $\mathrm{C}-\mathrm{H}$ atom prior the accommodation of molecular $\mathrm{O}_{2}$ and further construction of the $\mathrm{C}-\mathrm{O}$ double bond. 


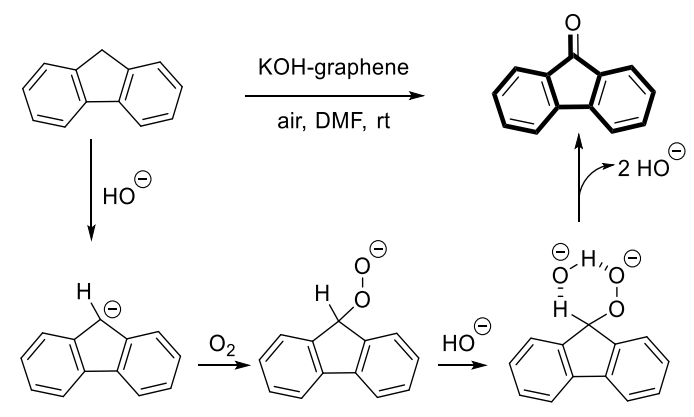

SCHEME 1 - Mechanism using $\mathrm{KOH}$-graphene as oxidant

Oxidation using molecular $\mathrm{O}_{2}$ under homogeneous basic conditions $\left(\mathrm{KOH}\right.$ or $\left.\mathrm{K}_{2} \mathrm{CO}_{3}\right)$ in the presence of 18-crown- 6 in DME at $100^{\circ} \mathrm{C}$ was also reported and allowed to obtain $17 \mathrm{H}$-tetrabenzo[a,c,g,i]fluorene in $60 \%$ yield. ${ }^{16}$ The ability of sodium bismuthate in refluxing acetic acid/acetone/water mixtures to efficiently promote the oxidation at benzylic sites was recently reported by Becker ${ }^{17}$. This methodology was successfully applied to tetracyclic and pentacyclic fluorenes. Finally, bi-angular pentacyclic fluorene was reacted in the presence of triton-B in pyridine to give the corresponding polycyclic fluorenone in a quantitative yield. ${ }^{18}$

\section{To extended fluorenones through ring contraction}

The preparation of benzo[b]fluorenone has been described in two steps from benzoanthracenediones by Mal et al. (Scheme 2). ${ }^{19}$ This methodology is based on a base-promoted ring contraction. A benzylbenzylic acid rearrangement accounts for the first step leading to the $\alpha$-hydroxycarboxylic acid. The latter was then subjected to oxidative decarboxylation with $\mathrm{CrO}_{3}$ to afford several benzo[ $b$ ]fluorenones in $42-57 \%$ yield, depending on substitution of ring $C$.

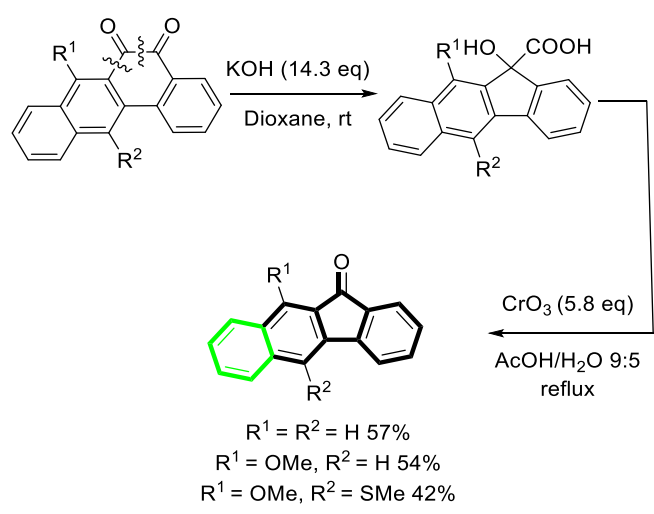

SCHEME 2 - Ring contraction using $\mathrm{KOH}-\mathrm{CrO}_{3}$ 
This section will focus on methods involving the construction of the central cyclopentanone ring as the key step in the preparation of the extended fluorenone scaffold.

\section{4-1. Construction of ring A from diarylketones}

The construction of extended fluorenones was first considered from 2-benzoylnaphthalene derivatives. In these cases, the construction of a $C_{\mathrm{sp} 2}-C_{s p 2}$ bond between both aromatic fragments allows for the formation of the central ring $A$ (Scheme 3 ). A route to extended fluorenones was also described using one pot- two-step procedures starting from separate benzene and naphthalene derivatives.



SCHEME 3 - Construction of ring A

One of the most efficient ways to prepare tetracyclic fluorenones is based on a double $\mathrm{C}-\mathrm{H}$ activation followed by reductive elimination to form the cyclopentanone unit. Both homogeneous and heterogeneous conditions have been developed involving $\mathrm{Pd}\left(\mathrm{OCOCF}_{3}\right)_{2} / \mathrm{Ag}_{2} \mathrm{O},{ }^{20} \mathrm{Pd}(\mathrm{OAc})_{2} / \mathrm{Ag}_{2} \mathrm{O}^{21}$ and $\mathrm{Pd} / \mathrm{Mg}$-La mixed oxide ${ }^{22}$ as catalytic combinations. In all cases, a six-membered palladacycle intermediate is formed prior to ring closure. Site selective $\mathrm{C}-\mathrm{H}$ insertion of the palladium is crucial in determining the shape of the product. Indeed, $\mathrm{C}-\mathrm{H}$ insertion at the naphthalene fragment might occur at both $\mathrm{C} 1$ and $\mathrm{C} 3$ sites leading to the formation of benzo[c]- or benzo[b]fluorenones, respectively. In fact, the benzo[b]fluorenone is selectively produced in good agreement with intrinsic reactivity of naphthalene substrates ${ }^{21}$ and general observations in $\mathrm{C}-\mathrm{H}$ functionalization processes in the naphthalene series (Scheme 4). ${ }^{23,24}$ 

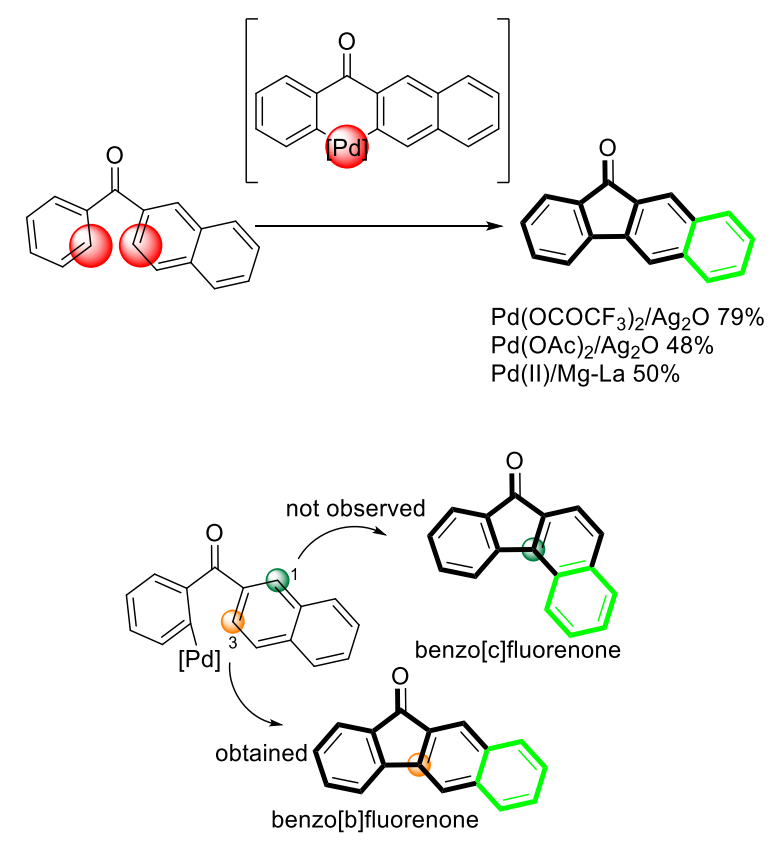

SCHEME 4 - Site selection at naphthalene substrates

The one-pot two-step sequences independently developed by Dharmaraj ${ }^{25}$ and Chikhalia ${ }^{26}$ (Scheme 5) represent further advances in which the 2-benzoylnaphthalene derivative is produced in situ from the combination of benzoyl chloride and naphthalene boronic acid on one hand and naphthalene carboxaldehyde and ortho-chloro iodobenzene on the other hand.

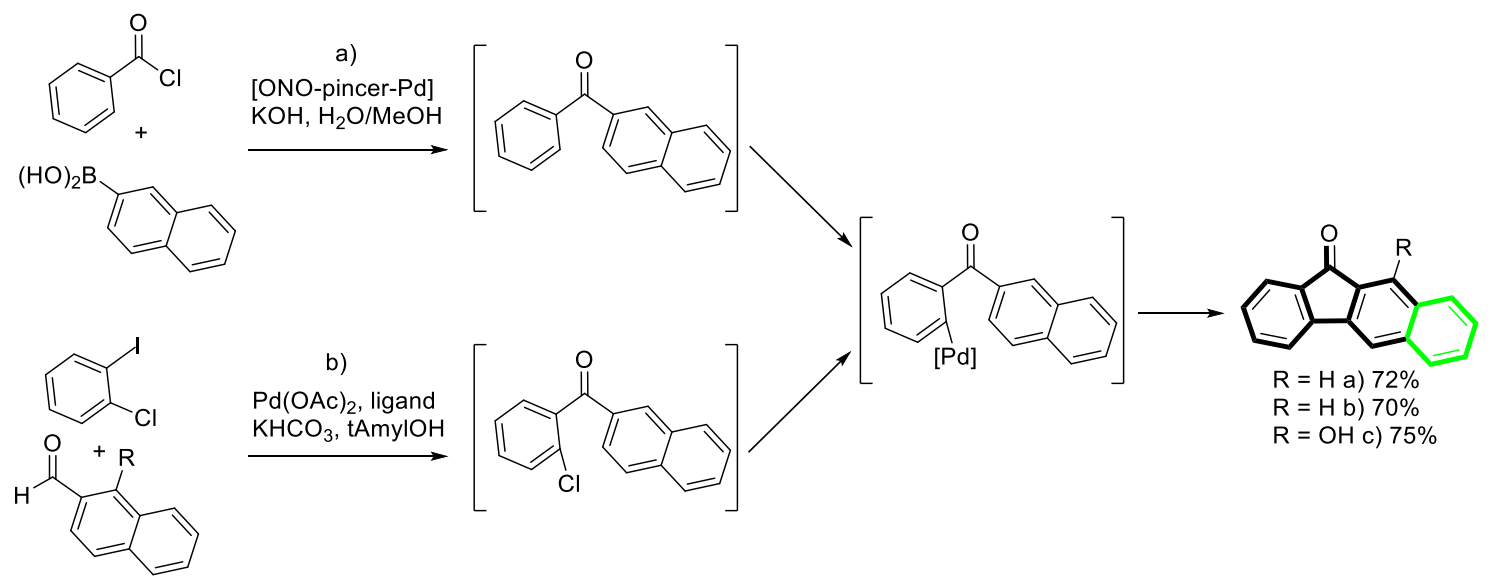

SCHEME 5 - One-pot two-step sequences leading to extended fluorenones

Both Pd-catalyzed first steps result in the formation of 2-benzoylnaphthalene intermediates which only differ by the presence of an ortho chloride atom. The subsequent Pd-complex is either obtained through $\mathrm{C}-\mathrm{H}$ insertion (a) or oxidative addition (b). The final fluorenone derivatives are thus most likely obtained through a six-membered palladacycle.

Alternative scenarios involving dibromobenzylalcohols or radical processes have been reported. Interestingly, both are dedicated to the selective formation of benzo[c]fluorenones. As highlighted above, the site selection in $\mathrm{C}-\mathrm{H}$ functionalization processes generally favors the $\mathrm{C} 3$ site at the naphthalene. In order to overcome this hurdle, the following pathway implies a bromine atom located at $\mathrm{C} 1$ (Scheme 6). ${ }^{27}$ The overall sequence involves a benzyl-naphthyl precursor bearing two bromine 
atoms. After examination of several plausible mechanisms, authors stated that the formation of the target most probably involves a palladium-catalyzed oxidation of the secondary alcohol prior to an intramolecular reductive coupling of $\mathrm{C}-\mathrm{Br}$ bonds.

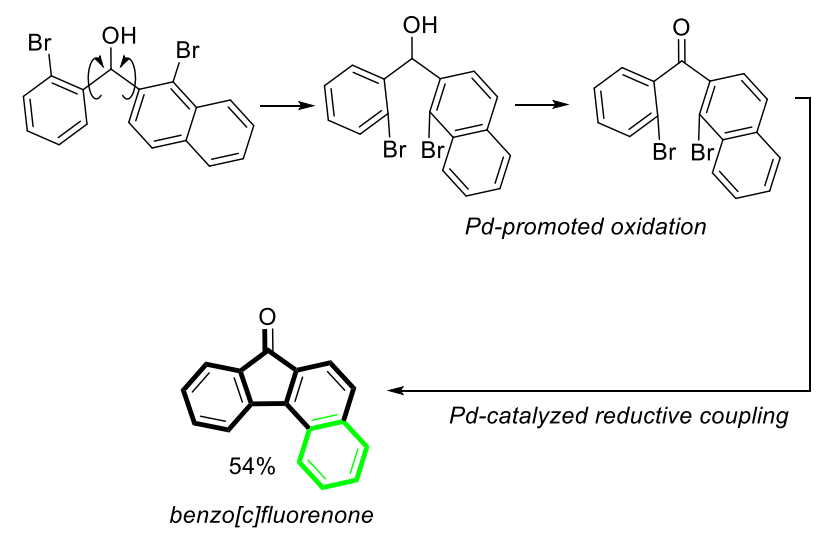

SCHEME 6 - Synthesis of a benzo[c]fluorenone from a dibromobenzylalcohol through a Pd-catalyzed reductive coupling

A similar approach using a radical decarboxylation arylation-based transformation has also allowed the formation of benzo[c]fluorenone. ${ }^{28}$ In this case the presence of a carboxylic acid group located at C1 site of the naphthalene fragment triggered the oxidative decarboxylation, generating a radical species at the $\mathrm{C} 1$ site using $\mathrm{Ag}^{+} / \mathrm{K}_{2} \mathrm{~S}_{2} \mathrm{O}_{8}$ which subsequently led to ring closure. Finally, a further second one-electron oxidation and proton loss is responsible for the selective transformation towards benzo[c]fluorenone in $31 \%$ yield (Scheme 7).

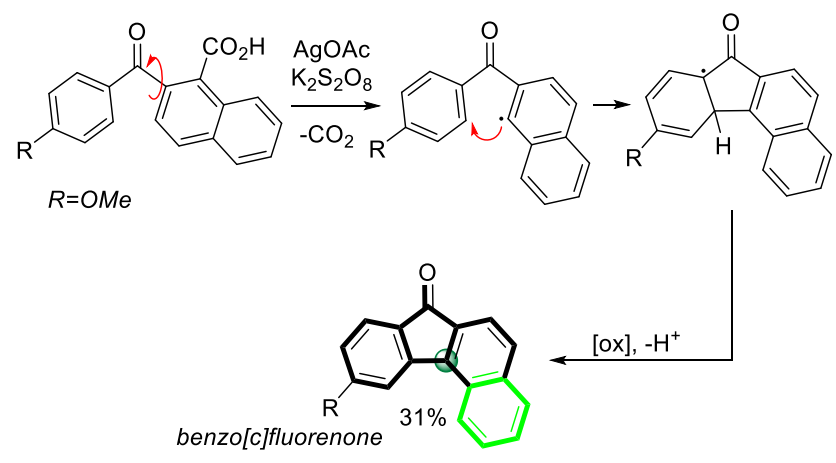

SCHEME 7-Synthesis of a benzo[c]fluorenone through a radical process from an alternative substrate 


\section{4-2. Construction of ring A from ortho functionalized biaryl precursors}

The construction of the fluorenone scaffold has also been proposed from biaryl starting materials(Scheme 8). With the aim to obtain tetra- and pentacyclic architectures, substituted phenylnaphthalenes and binaphthyls have been mainly considered as valuable precursors. This section is organized around the nature of the functional group.



SCHEME 8-Construction of ring A

We first focus on the carboxaldehyde moiety. In this context, Glorius explored a radical cyclisation process towards the formation of benzo[c]fluorenones. ${ }^{29}$ The authors used tetraethylammonium bromide(TEAB)/ $\mathrm{K}_{2} \mathrm{~S}_{2} \mathrm{O}_{8}$ as the radical generating combination to produce the acyl radical intermediate (Scheme 9). Annulation followed by oxidation and deprotonation allowed the formation of the fluorenone architecture from naphthalene and phenanthrene precursors in 58 and $62 \%$ yields, respectively.

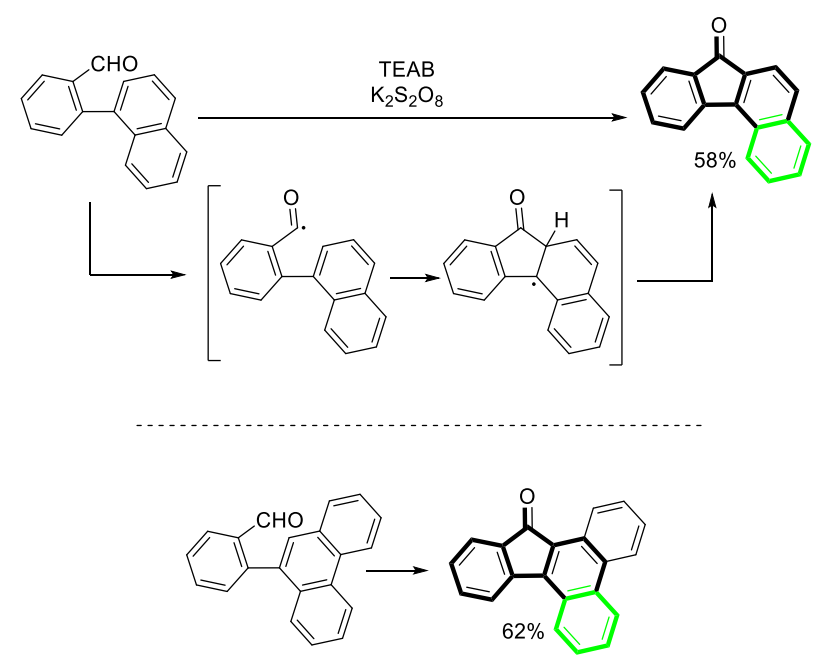

SCHEME 9 - Use of TEAB for the construction of ring A

In addition, Pd-promoted reactions of ortho bromo carboxaldehydes and boronic acids have been reported enabling the access to diversely substituted extended fluorenones. This one-pot process affords benzo[ $a]$ - and benzo[c]fluorenones depending on the substitution pattern of the naphthalene reactant. In the presence of $\mathrm{Pd}(\mathrm{OAc})_{2}, \mathrm{NaOAc}$ and $\mathrm{PPh}_{3}$, benzhydryl alcohols are first produced, followed by annulation and finally oxidation towards the fluorenone scaffold. ${ }^{30}$ In the fluorenone series, in which only benzene derivatives are used as starting material, the presence of two halides is not required to ensure annulation. However, in the naphthalene series the use of substrates both bearing a $\mathrm{Br}$ atom is mandatory to selectively generate benzo[ $a]$ - or benzo[c]fluorenones (Scheme 10 ). 

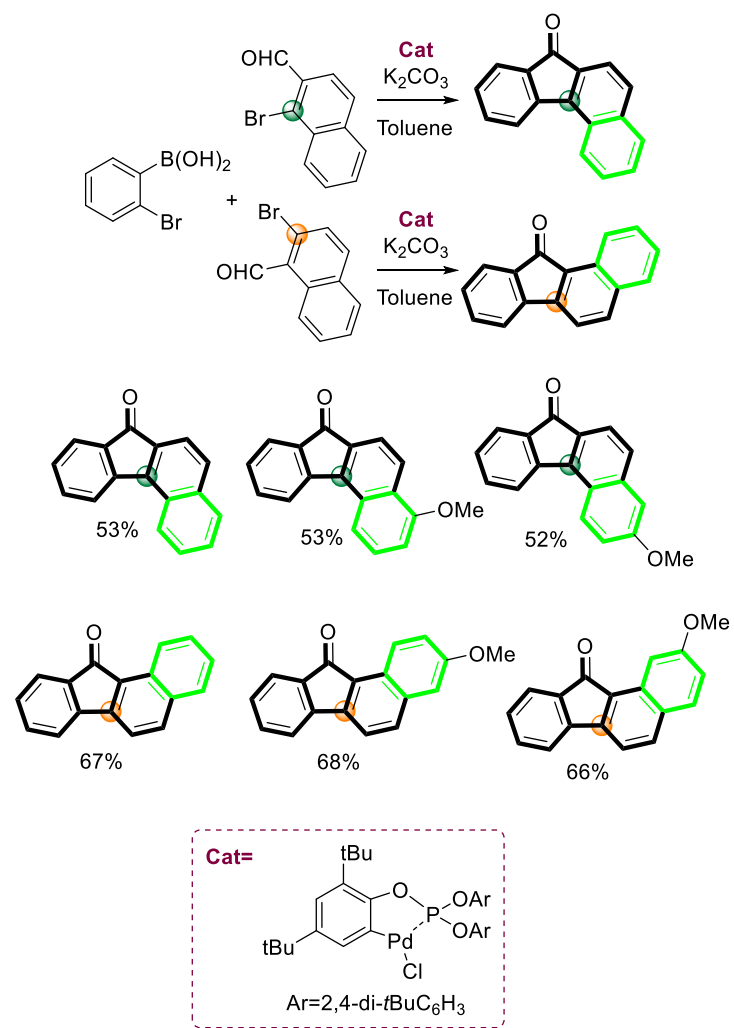

SCHEME 10 - From o-bromo carboxaldehydes and boronic acids to extended fluorenones

Interestingly this strategy is relevant for the selective installation of substituents at the naphthalene fragment of both benzo[a]- or benzo[c]fluorenones. A judicious alternative, developed by Lautens et al. using 1-idodo-naphthalene and variously substituted ortho-bromobenzaldehydes as reagents, allows access to the benzo[a]fluorenones substituted at the opposite benzene fragment. ${ }^{31}$ The pathway implies a domino ortho-arylation and a subsequent addition to the carbonyl group. The norbornene mediated $\mathrm{C}-\mathrm{H}$ activation at the 1-iodonaphthalene substrate is a key step, determining the selective formation of the $\mathrm{C}-\mathrm{C}$ bisaryl bond at position 2 of the naphthalene unit (Scheme 11).

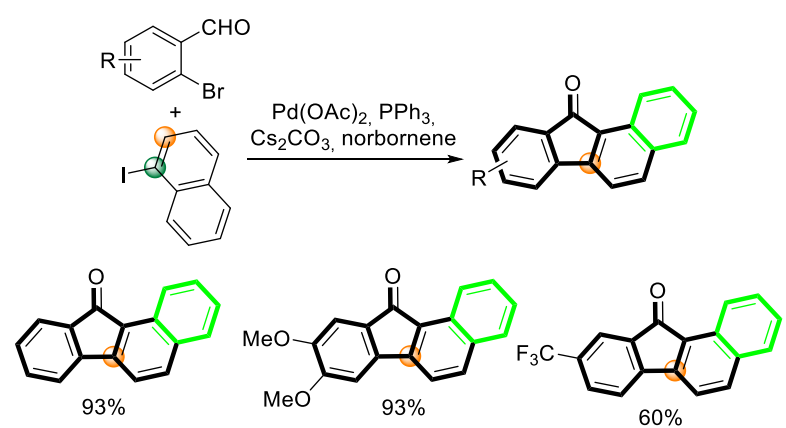

SCHEME 11 - From o-bromo carboxaldehydes and iodonaphthalenes to extended fluorenones

Within these series of substituted biaryl substrates, imines and oximes have emerged as valuable precursors of carbonyl moiety. Again, $\mathrm{Pd}$-catalyzed and metal free procedures have been developed to prepare extended fluorenones. The use of hypervalent iodine ${ }^{32}$ or $\mathrm{Pd}(\mathrm{OAc})_{2}$, dppm, $\mathrm{CsPiv}^{33}$ represent elegant and selective alternatives to carboxaldehydes towards extended fluorenones. It is worth noting that both processes result in the late formation of imines, whose hydrolysis allows to recover the fluorenone derivative(Scheme 12). 

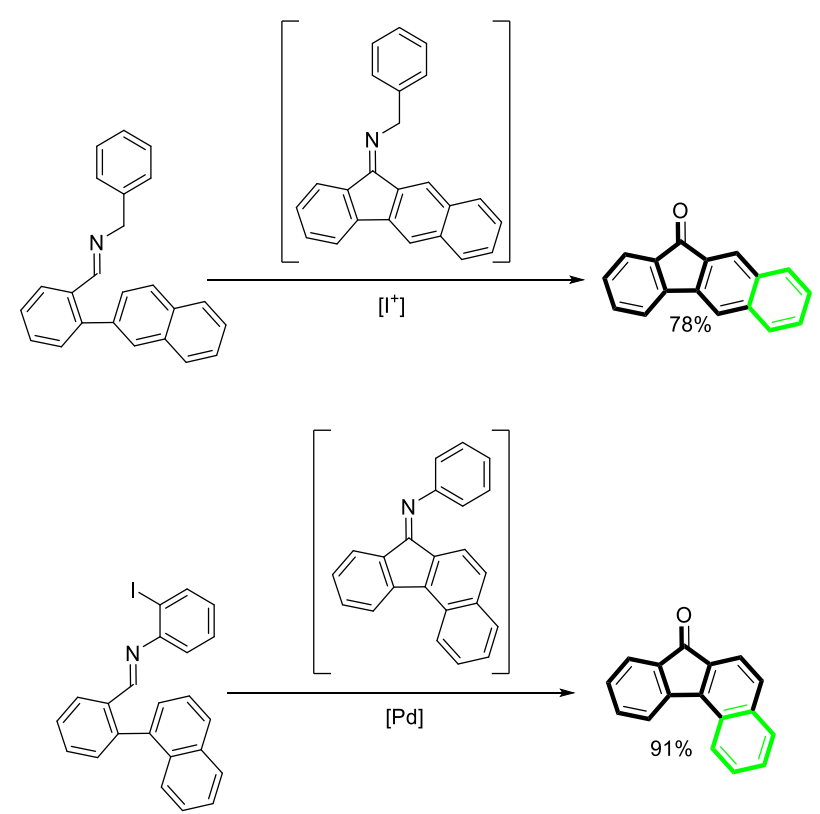

SCHEME 12 - Hydrolysis of imines leading to the formation of ring A

Recently, 2-halogenated biphenyls have been employed as starting materials for the preparation of extended fluorenones. In this palladium-catalyzed protocol, phenyl formate acts as a carbon monoxide surrogate (Scheme 13). ${ }^{34}$ As a key step, carbon monoxide is generated from the reaction between phenyl formate and an arylcarboxylate. The latter inserts into the catalytic cycle affording a cyclic acylpalladate which undergoes reductive elimination towards the desired extended fluorenone.

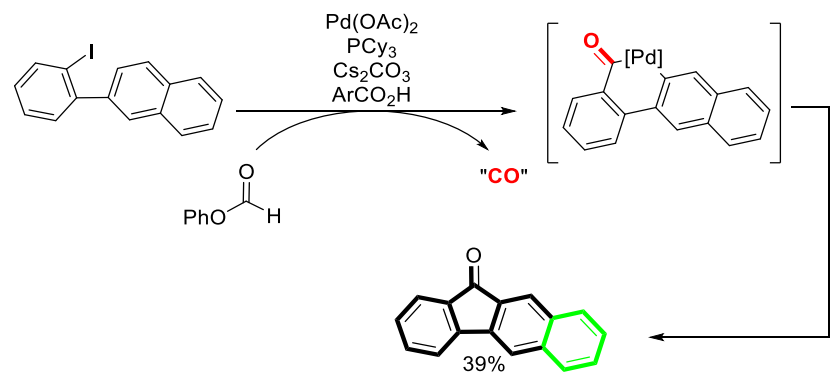

SCHEME 13-Use of phenyl formate as a CO surrogate

A further approach consists of a one pot formation of the biarylprecursor and cyclisation towards fluorenones (Scheme 14). As described by Cheng, ${ }^{35,36}$ oximes can act as ortho metalation directing group. A domino process involving two iterative $\mathrm{Pd}$-catalyzed $\mathrm{C}-\mathrm{H}$ insertions account for the formation of phenylnaphthalene platform and the construction of the cyclopentanone unit. The first $\mathrm{C}-\mathrm{H}$ functionalization allowed the installation of a phenyl substituent at position 3 of the naphthalene unit. The subsequent $\mathrm{C}-\mathrm{H}$ functionalization is devoted to carbopalladation of the oxime $\mathrm{C}-\mathrm{N}$ double bond. Finally, hydrolysis in acidic conditions generates the cyclopentanone fragment. 

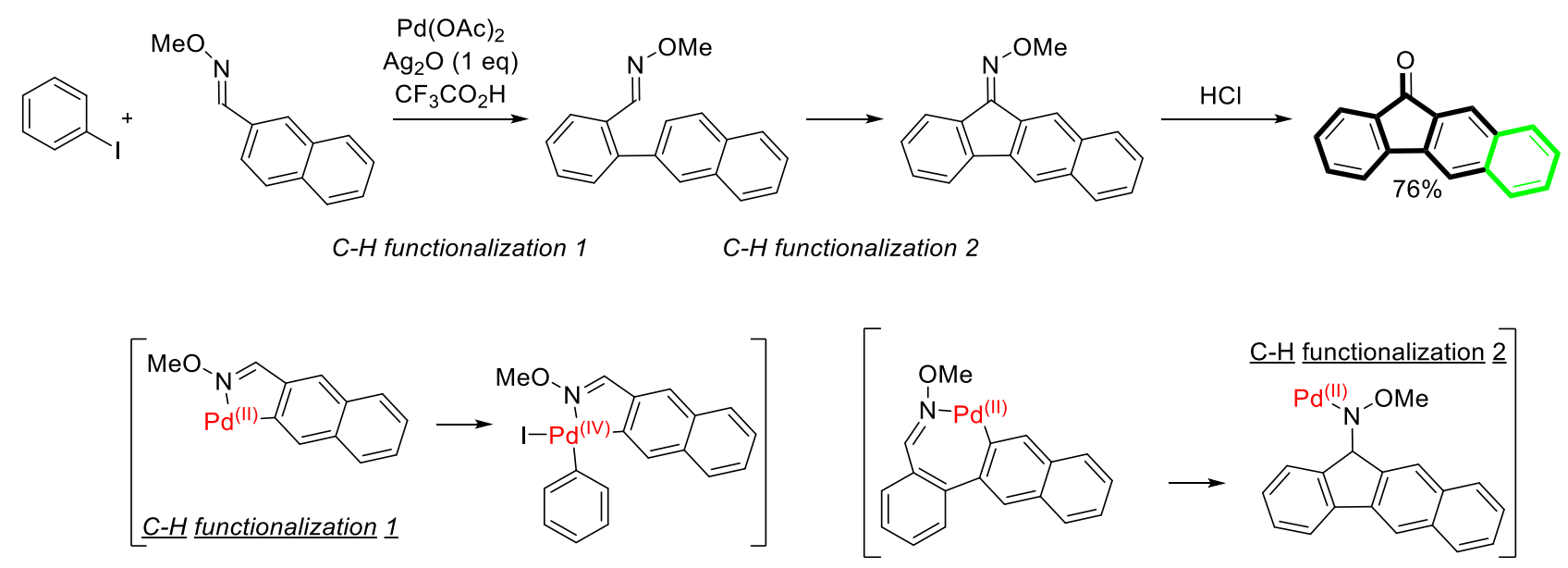

SCHEME 14 - Domino process described by Chen

The construction of ring A from biaryl precursors bearing carboxylic acid derivatives is one of the historical and most prolific ways to access fluorenones. Two main ways are reported through electrophilic aromatic substitution using either Brönsted acids or metal-promoted transformations.

In this context, rhodium-catalyzed intramolecular acylations of biarylcarboxylic acids have been successful (Scheme 15), leading to the benzo[c]fluorenone in $93 \%$ yield. ${ }^{37}$ The acyl rhodium species acts as a key intermediate which is formed first and then undergos an intramolecular $\mathrm{C}-\mathrm{H}$ acylation to afford the rhodacycle. The target, an extended fluorenone is finally produced through reductive elimination.

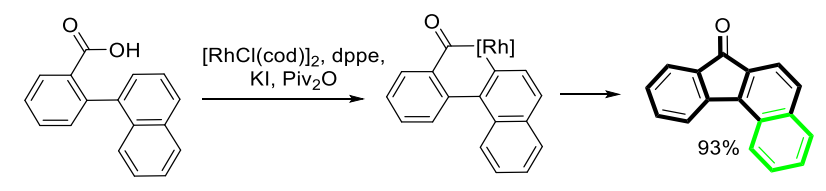

SCHEME 15 - Rhodium-catalyzed intramolecular acylations of biarylcarboxylic acids

Photoredox catalysis has also been used to initiate the formation of fluorenones. ${ }^{38}$ In this approach, a photocatalyst, fac-Ir(ppy) $)_{3}$ in the presence of dimethyl dicarbonate (DMDC) under blue LED irradiation induces the formation of an acyl radical from a carboxylic acid moiety. The acyl radical is assumed to undergo intramolecular addition to the naphthalene fragment affording the benzo[c]fluorenone in $67 \%$ yield (Scheme 16$)$.

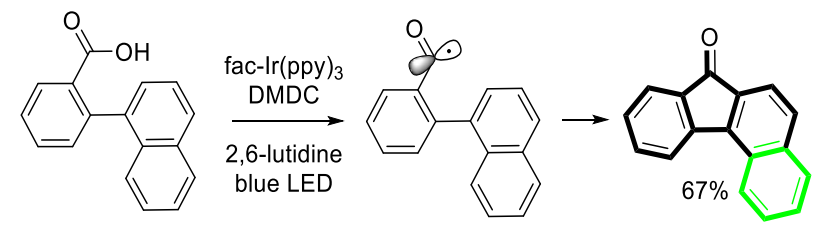

SCHEME 16 - Photo redox catalysis

Recently, You has reported an elegant strategy towards fluorenones involving a Pd-catalyzed selective oxidative $\mathrm{C}-\mathrm{H} / \mathrm{C}-\mathrm{H}$ cross-coupling of aromatic carboxylic acids with arenes. ${ }^{39}$ This strategy affords a straightforward route to the diaromatic carboxylic acid using $\mathrm{Pd}(\mathrm{OAc})_{2}, \mathrm{Ac}-\mathrm{Ile}-\mathrm{OH}, \mathrm{Na}_{2} \mathrm{~S}_{2} \mathrm{O}_{8}$ as the catalytic combination. The latter is obtained via ortho $\mathrm{C}-\mathrm{H}$ insertion, formation of the palladacycle, reaction with benzene and after reductive elimination (Scheme 17). A subsequent intramolecular 
Friedel-Crafts acylation under acidic conditions ( $\mathrm{TfOH})$ affords the expected extended fluorenone in $53 \%$ yield.



SCHEME 17 - Pd-catalyzed selective oxidative $\mathrm{C}-\mathrm{H} / \mathrm{C}-\mathrm{H}$ cross-coupling of aromatic carboxylic acids with arenes

Among synthetic strategies which have been reported for the construction of extended fluorenones, Friedel-Crafts acylation of biarylcarboxylic acids and their derivatives is the most convenient. The obtention of extended fluorenones from these precursors have been described using Lewis acids such as $\mathrm{BCl}_{3}$, as reported by Torres et al. ${ }^{40}$ during their study of an elegant one-pot synthesis of $\pi$-extended fluorenone-fused subphthalocyanines. These impressive chromophores were obtained from from simple reactants and were extensively studied in the same report. However, in majority of the cases, cyclization is induced by Bronsted acids such as sulfuric acid, methane sulfonic acid, and polyphosphoric acid (PPA), and follows a mechanism involving an acylium and a Wheland intermediate. Since the late 19th century, sulfuric acid has been known to promote the cyclisation of a carboxylic acid onto a naphthalene moiety, leading to the formation of an extended fluorenone. ${ }^{41}$ Subsequently, $\mathrm{H}_{2} \mathrm{SO}_{4}$ has been used by multiple times over the years ${ }^{42}$ and was almost used exclusively to perform this reaction until Osowiecki et al. first introduced PPA to do similar cyclisation reactions. ${ }^{43}$ PPA quickly became one of the most common acids to perform such reaction. ${ }^{44}$ It was notably used by Brown ${ }^{45}$ who showed that this cyclization reaction was reversible using $\mathrm{KOH}$ followed by PPA to successively break and reform ring A of an extended fluorenone by Gould's group, who showed this reaction was also working on esters ${ }^{46}$ and by Skibo's team to synthesize ${ }^{13} \mathrm{C}$ marked fluorenones for biological studies. ${ }^{47}$

Sulfonic acids such as HOTf are being used more frequently to promote such reactions, as shown by Zhu's group in a single example. ${ }^{48} \mathrm{HOTf}$ and $\mathrm{MsOH}$ have then been used several times to promote the formation of extended fluorenone in the field of optoelectronics by Frigoli's team ${ }^{49}$ and Park's group (Scheme 18). ${ }^{50}$ 


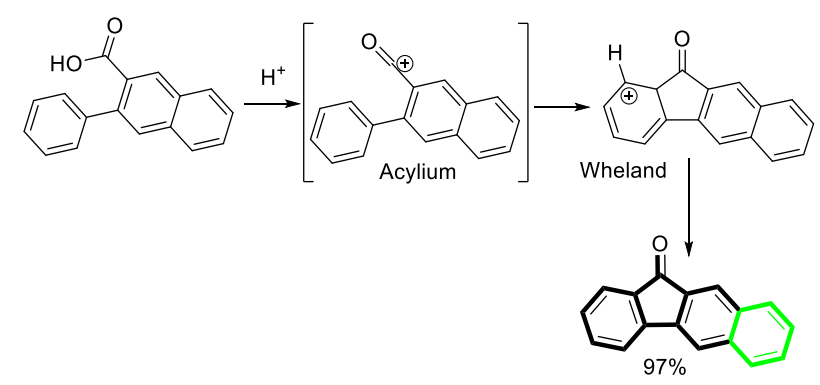

SCHEME 18 - Friedel-Craft acylations of carboxylic acids

Electrophilic cyclisation has also been described from $\mathrm{N}$-tosyl naphthamides leading to tetra- and pentacyclic fluorenones (Scheme 19). Originally exemplified once in the fluorenone series ${ }^{51}$, the $\mathrm{N}$ tosylamide group was more extensively studied as acylium precursors in the construction of extended fluorenones..$^{32,33}$ Our contribution revealed that the $N$-tosylamide group was especially helpful as a directing group for the selective $\mathrm{C}-\mathrm{H}$ arylation of naphthalene substrates. A further advantage lies in the possible generation of acylium moieties under acidic conditions ( $\mathrm{TfOH}, \mathrm{AcOH}$ ) and construction of the central ring A. Both benzo[b]- and benzo[a]fluorenones could be obtained depending on the substitution pattern of the starting material (Figure 4). The benzo[b] moiety is obtained from substrates bearing the $\mathrm{N}$-tosylamide at position two of the naphthalene precursor. In contrast, benzo[a]- analogues are formed from substrates bearing the $N$-tosylamide at position one. Unequivocal cyclisation occurs in each case for symmetrically substituted ring B.

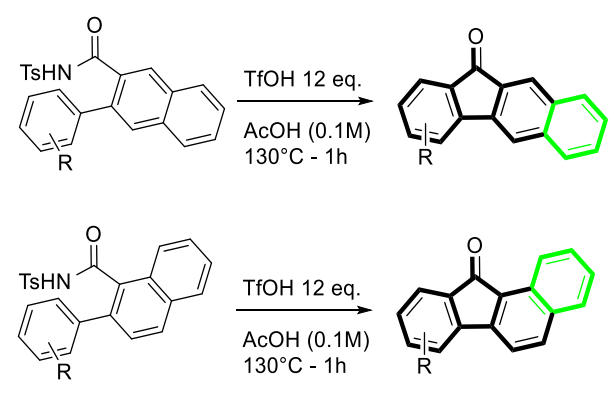

SCHEME 19 - Friedel-Crafts acylation of amides 



FIGURE 4 - Scope of the Friedel-Crafts acylation of amides

In this article, the regioselectivity of the cyclization and discrimination between the angular and the linear isomers was also studied for the first time through DFT calculations (Figure 5). When the starting material displays two different cyclisation sites, mixtures of products might be observed in various ratios depending on the substitution pattern of the arylated naphthalene and both on the steric crowding and electronic factors. Several tetracyclic and pentacyclic extended fluorenones were synthesized using this pathway and strategy.
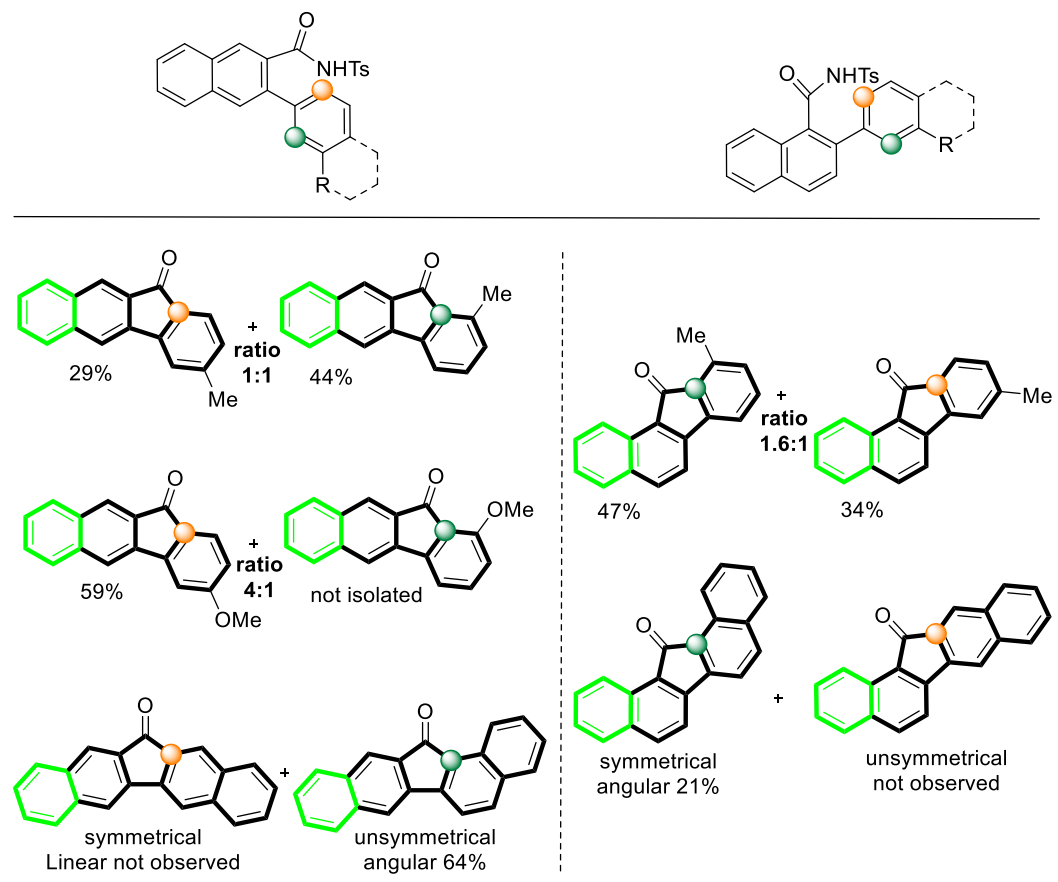

FIGURE 5 - Selectivity of the Friedel-Crafts acylation of amides

An elegant 2-step domino process was described in 2009 by Lautens' team starting from ester derivative precursor of the fluorenone carbonyl moiety: the combination of a Catellani reaction 
followed by a pallado catalyzed $\alpha$-addition on a carbonyl has allowed the construction of 2 new bonds and the cycle $A$ of several fluorenones. This methodology was also used to synthesize reduced fluorenones and phenanthrene derivatives in very good yields (Scheme 20). ${ }^{52}$

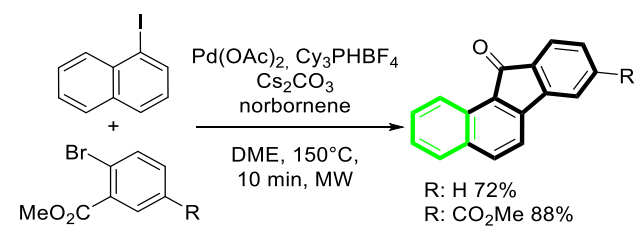

SCHEME 20 - Two-step domino process from Lautens

According to Kubo the doubly benzo[c]-condensed fluorenone was also reported (Scheme 21). ${ }^{53}$ The pentacyclic target was obtained from a binaphthyl bis carboxylic acid as the starting material utilizing rather harsh reaction conditions involving acetic anhydride at up to $300^{\circ} \mathrm{C}$, and delivering the expected product with a medium-low yield (37\%).

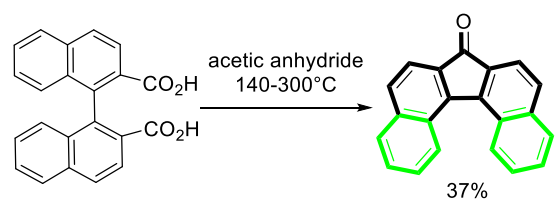

SCHEME 21 - Double condensation

A straightforward pathway to tetra and pentacyclic fluorenones has been recently reported using cobalt-catalyzed MHP-directed [3+2] annulation/ ring-opening/dehydration sequence (Scheme 22)..$^{54}$ This methodology is not only especially adapted to extended fluorenones but also allows to access a broad scope of substituted targets. In this protocol, molecular $\mathrm{O}_{2}$, as the green oxidant, is mandatory

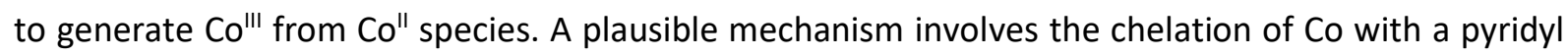
hydrazide derivative and a subsequent ortho $\mathrm{C}-\mathrm{H}$ activation. Coordination of the oxabicyclic starting material followed by migratory insertion allowed the formation of the seven-membered cobaltacycle. The latter undergoes a ring contraction towards the cyclopentane core. The loss of pyridyl hydrazine results in the formation of the ketone moiety. The presence of $\mathrm{Cs}_{2} \mathrm{CO}_{3}$ promoted the ring opening and final dehydration steps lead to the benzo[b]fluorenone. The protocol was also shown to be efficient for the preparation of pentacyclic fluorenones (Figure 6). 


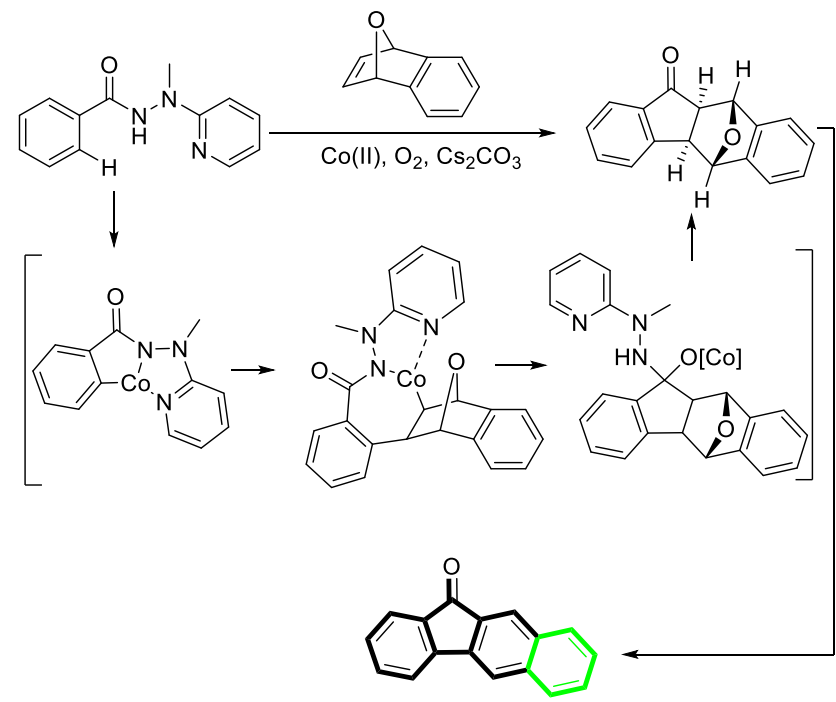

SCHEME 22 - Use of cobalt for the preparation of pentacyclic fluorenones

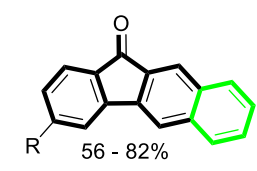

$\mathrm{R}=\mathrm{H}, \mathrm{Me}, \mathrm{OMe}, \mathrm{OPh}, \mathrm{OCF}_{3}$, ${ }^{t} \mathrm{Bu}, \mathrm{SMe}, \mathrm{Ph}, \mathrm{Cl}, \mathrm{Br}, \mathrm{I}, \mathrm{CF}_{3}$

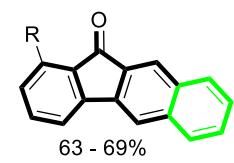

$\mathrm{R}=\mathrm{Me}, \mathrm{OMe}, \mathrm{Cl}$
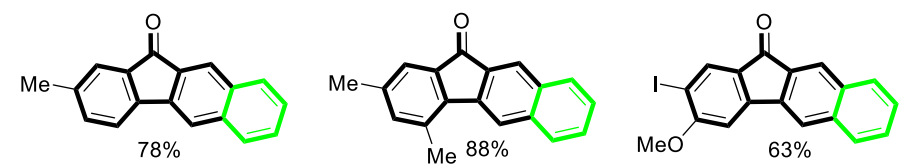
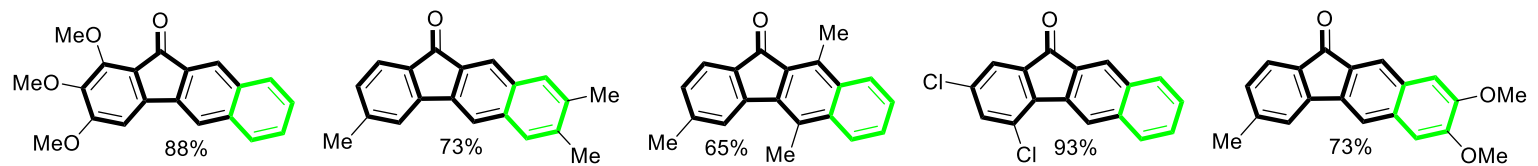

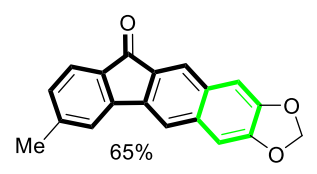<smiles></smiles>
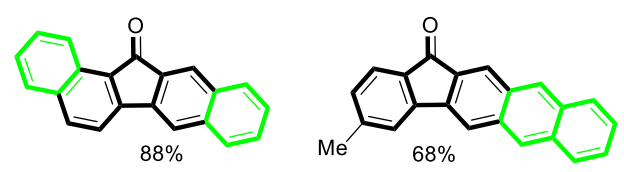

FIGURE 6 - Scope of the cobalt-promoted preparation of pentacyclic fluorenones

The construction of ring A from biaryl precursors can also be achieved from cyanide precursors. Both metals promoted protocols and electrophilic aromatic substitutions under Brönsted acidic conditions have been reported. In the $\mathrm{Pd}$-catalyzed process, the nitrile group undergoes directed remote $\mathrm{C}-\mathrm{H}$ bond activation. Subsequent 1,2-insertion of nitrile is responsible for the formation of the imine-type intermediate which is then hydrolyzed by $\mathrm{H}_{2} \mathrm{O}$ to provide the desired benzo[c]fluorenone (Scheme 23). ${ }^{55}$ Also a one-pot process from separate aryl precursors has been reported in the tricyclic $9 \mathrm{H}$ fluoren-9-one series, however, it has not been applied in higher homologues. As already mentioned in this review, a similar process involving halogenated substrates represent an alternative for reaching different selectivities. In this context, the desired benzo[b]fluorenone could be obtained from iodocyano substrates in $51 \%$ yield. ${ }^{56}$ 


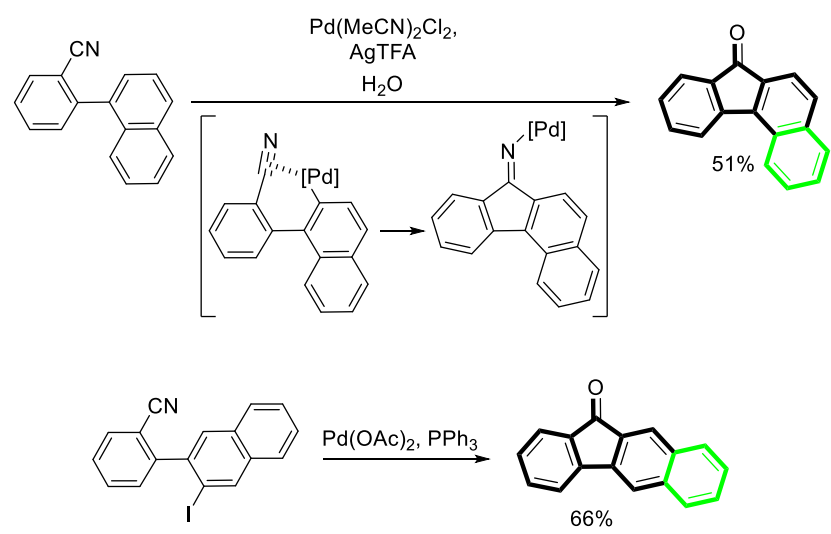

SCHEME 23 - Pd-catalyzed construction of Ring A from cyanides

Nitriles are known precursors of ketones when treated in strong acidic media. The syntheses of several benzo[b]fluorenones has been described using electrophilic aromatic substitutions from nitrile derivatives. In this context, PPA (polyphosphoric acid) at $120^{\circ} \mathrm{C}$ revealed often superior to other Brönsted acids. ${ }^{57}$ Under such acidic conditions, tetracyclic but also penta- and hexa-cyclic fluorenones could be isolated either from binaphthyl-type starting material or from precursors based on phenanthryl residues. Comparison of $\mathrm{CN}$ and esters groups was also done under PPA-promoted conditions (Scheme 24). ${ }^{58}$ Interestingly, cyclisation towards the fluorenone scaffold was more efficient using nitrile as an electrophile precursor than the ester group. ${ }_{-}^{59}$



SCHEME 24 - PPA induced construction of Ring A from cyanide

Construction of ring $\mathrm{A}$ of fluorenones has been recently described from cyclic diaryliodonium salts under Pd-catalyzed carbonylation conditions (Scheme 25). ${ }^{60}$ 




SCHEME 25 - Construction of ring A from cyclic diaryliodonium salts

The overall process begins with $\mathrm{Pd}^{0}$ induced ring opening of cyclic diaryliodonium salt. The resulting complex undergoes successive $\mathrm{CO}$ insertion, followed by oxidative addition into the neighboring $\mathrm{C}-\mathrm{I}$ bond and finally reductive elimination resulting in the desired ketone. 


\section{4-3. Construction of ring A from aryne precursors}

The presence of the fluoren-9-one scaffold in natural products such as dengibsin, dengibsinin and dendroflorin, has led to the development of new synthetic routes to this family of polycyclic architectures. In this context, Larock et al. focused on Pd-catalyzed annulation reaction between in situ generated arynes and 2-haloarenecarboxaldehydes (Scheme 26). ${ }^{61,62}$ In the first step, arynes are generated in situ from 2-(trimethylsilyl)aryl triflates and CSF. Arynes then undergo annulation providing a useful new pathway to diversely substituted fluoren-9-ones and extended fluorenones in good yields. The procedure is well adapted to the pre-formation of the benzyne intermediate and naphtyl or phenanthryl reactants.
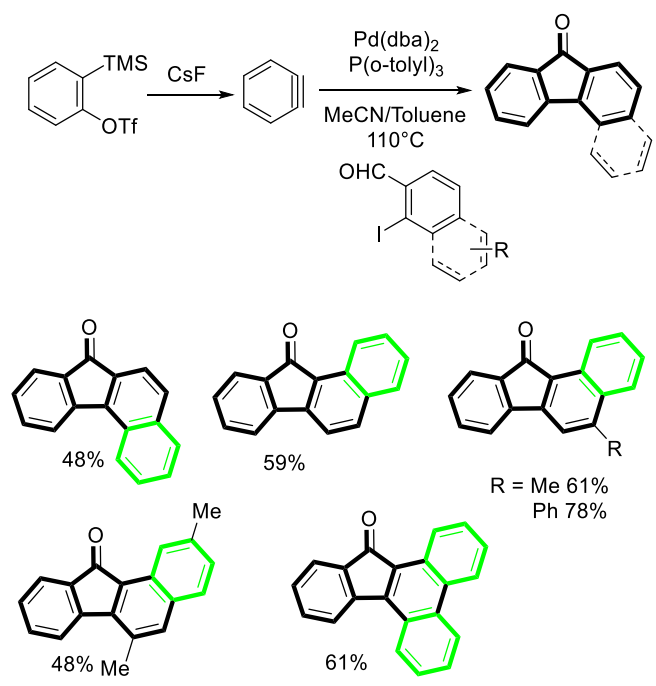

SCHEME 26 - From arynes to extended fluorenones

\section{To extended fluorenones through the successive construction of the rings $B$ and $C$}

An alternative appealing strategy towards pentacyclic fluorenones has been described by McLaughin et $a l . .^{63}$ The strategy involves two iterative Diels-Alder/rearomatization reactions starting from a cyclopentenone (Scheme 27). The latter undergoes a cycloaddition with tetrabromo-o-xylene and further rearomatization leading to the tricyclic benzo[f]indan-one. Bromination followed by dehydrohalogenation affords the benzo[f]inden-one which was subjected to a second DielsAlder/rearomatization route to yield the extended fluorenone in an overall $28 \%$ yield.

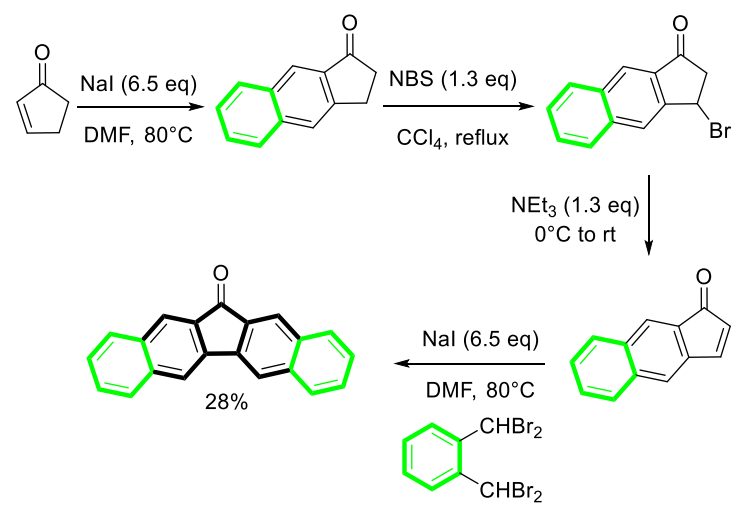

SCHEME 27 - Iterative Diels-Alder/rearomatization sequences 
Although this strategy has the advantage of being simple to implement and allows rapid access to a pentacyclic dibenzofluorenone, the molecular diversity of the final compound is greatly limited by the substitution of the reagent, which has been proved difficult to alter.

\section{To extended fluorenones through the joint construction of the rings $A$ and $C$}

Among methodologies for accessing the extended fluorenone structures, cycloadditions involving the joint construction of both ring $A$ and $C$, has proved to be a successful alternative. The presence of the tetracyclic core of benzo[b]fluorenone in natural products such as kinafluorenone, has prompted the development of highly selective cycloaddition-based pathways. In addition, these methologies allow for a wide modulation of the substitution pattern of rings $C$ and $D$.

The synthesis of tricyclic fluorenones through a [4+2] cycloaddition strategy was first described by Danheiser in 1994, ${ }^{64}$ however, since several other groups have expanded the scope of this transformation towards diversely substituted extended fluorenones. To this end, 1,6-enynes and 1,6diynes have been considered as valuable starting materials. Recently Tu et al. described a thermal intramolecular Diels-Alder reaction starting from 1,6-enynes (Scheme 28). ${ }^{65}$ The adduct is assumed to undergo 1,5 proton transfer leading to the fluorenol intermediate which subsequently converts into the expected fluorenone though a base-promoted oxidation.

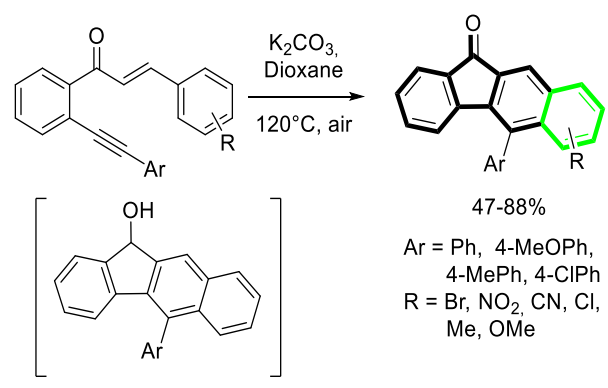

SCHEME 28 - Synthesis of tricyclic fluorenones through a [4+2] cycloaddition

Benzo[b]fluorenones have also been obtained from 1,6-diynes through a $\mathrm{Cu} / \mathrm{Selectfluor}$ mediated process (Scheme 29). ${ }^{66,67}$ The presence of Selectfluor appears to be necessary for the complete formation of the fluorenone but also in the electrophilic fluorination process which takes place with subsequent $\mathrm{C}-\mathrm{C}$ bond cleavage of the $t$-butyl group.

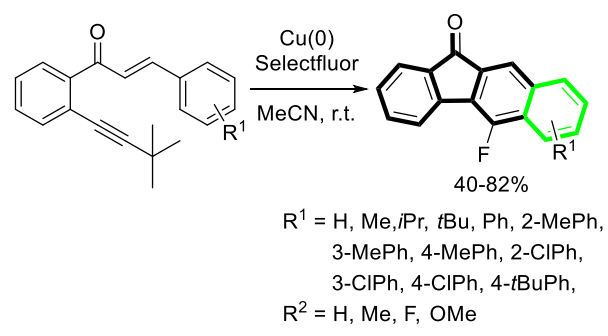

SCHEME 29-Obtention of Benzo[b]fluorenones from 1,6-diynes through a $\mathrm{Cu} /$ Selectfluor mediated process

First TMS-substituted diynes will be considered. In this context Echavarren ${ }^{6869}$ and Saá ${ }^{70}$ described the preparation of benzo[b]fluorenones under thermal neutral cycloaddition conditions (Scheme 30). 
Although the starting materials differ each other from the position of the TMS group, both pathways mainly afford mixtures of benzo[b]- and benzo[a]fluorenones or benzo[b]- and benzo[c]fluorenones.
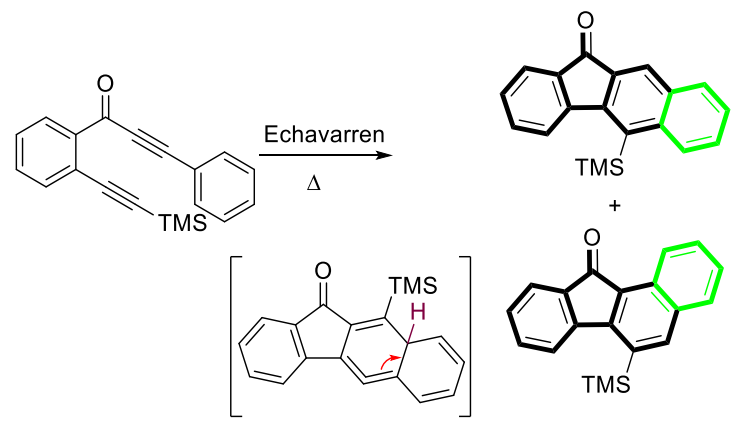

TMS
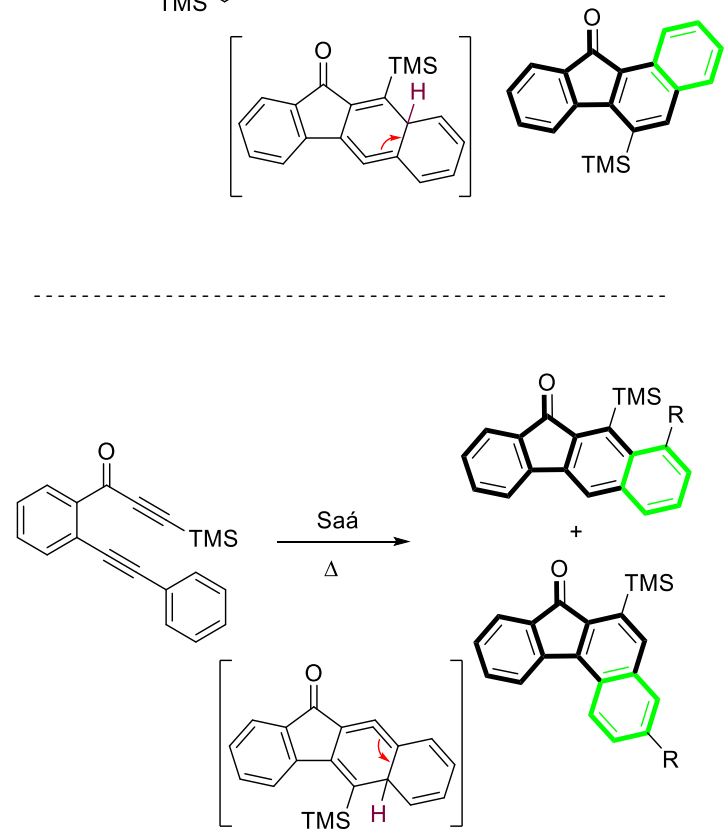

SCHEME 30 - Preparation of benzo[b]fluorenones from TMS-substituted diynes

In both cases, a mixture of benzofluorenones is obtained and its formation can be rationalized with a common intermediate, a strained allenic species. The latter undergoes a ring opening followed by ring closure towards either the benzo[a] or the benzo[c]fluorenones. Interestingly, Saá demonstrated that the benzo[b]- / benzo[c]fluorenone ratio is governed by electronic and steric factors and thus depends on the nature of the substituents. Indeed, the exclusive formation of the benzo[b]fluorenone is observed for hydroxyl and nitro groups. In contrast, large substituents such as $t$-butyl induce the exclusive formation of the benzo[c]fluorenone. In addition, under basic thermal conditions, Saá was also able to drive the reaction towards the formation of benzo[c]fluorenones (Scheme 31).

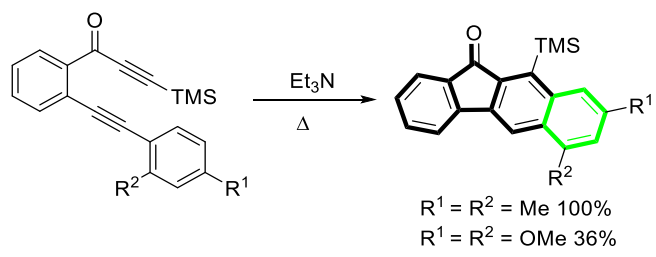

SCHEME 31 - Exclusive formation of benzo[c]fluorenone

More recently, Chen described an elegant Fe-catalyzed selective cycloaromatization of diynes (Scheme 32). The reaction involves the activation of the ketone moiety by an iron species and the subsequent formation of an allenyl nine-membered ring. The presence of NBS is required for the bromoniummediated construction of ring $\mathrm{C}^{71}$ The presence of a bromine atom has been shown useful for the subsequent installation of various aromatic fragments through Pd-Catalyzed reactions. This 
methodology has been demonstrated to be particularly useful for the preparation of aryl derivatives of benzo $[b]$ fluorenones $\left(\mathrm{R}^{3}=\mathrm{aryl}\right)$.



$\mathrm{R}^{1}, \mathrm{R}^{2}=\mathrm{H}, \mathrm{Me}, \mathrm{OMe}, \mathrm{F}, \mathrm{Cl}, \mathrm{Br}$

$\mathrm{R}^{3}=$ various aryl groups

key intermediates

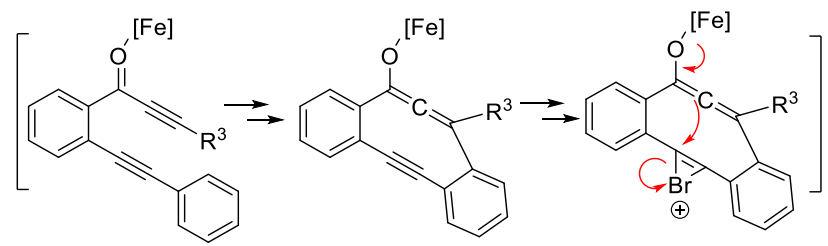

SCHEME 32 - Fe-catalyzed selective cycloaromatization of diynes

In a recent report, Balamurugan subjected similar 1,6-diyne starting materials bearing terminal aryl groups to Brönsted acid - promoted intramolecular cycloisomerization (Scheme 33). ${ }^{72}$ The procedure appears quite general and compatible with various substituents installed at all aromatic fragments of the reactants. As stated by the authors, $\mathrm{TfOH}$ is responsible for the protonation of the carbonyl group, facilitating the cyclisation into the indanone-type intermediate. The latter undergoes radical cyclisation to a strained allenic species which upon loss of $\mathrm{H}^{+}$or $\mathrm{H}$ affords the expected benzo[b]fluorenone.

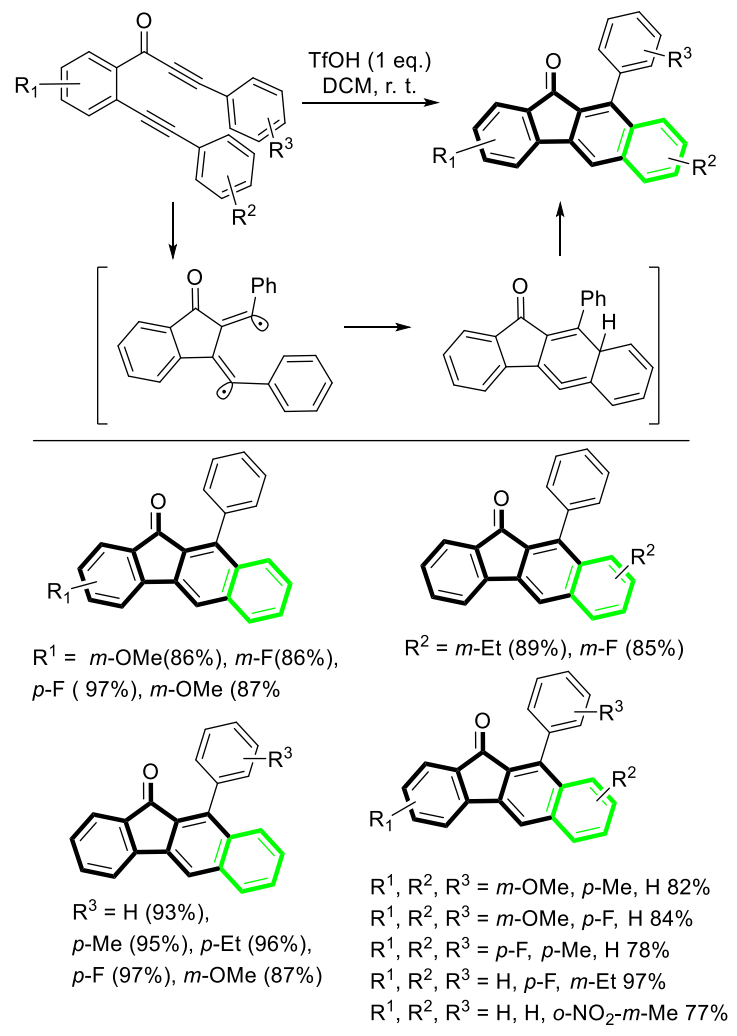

SCHEME 33 - Acid induced selective cycloaromatization of diynes 
Routes to benzo[b]fluorenones have also been reported from various types of starting materials. For example, 1,5-enynols and 1,5-diynols have been demonstrated to undergo cycloaromatization under oxidative conditions. ${ }^{73,74}$ DDQ-promoted cycloaromatization showed excellent functional group tolerance allowing the installation of various substituents including alkyl and ester groups. Interestingly, both methodologies have been proven to be especially fitted for the preparation of fluorenones bearing two different aryl groups located at ring C (Scheme 34).
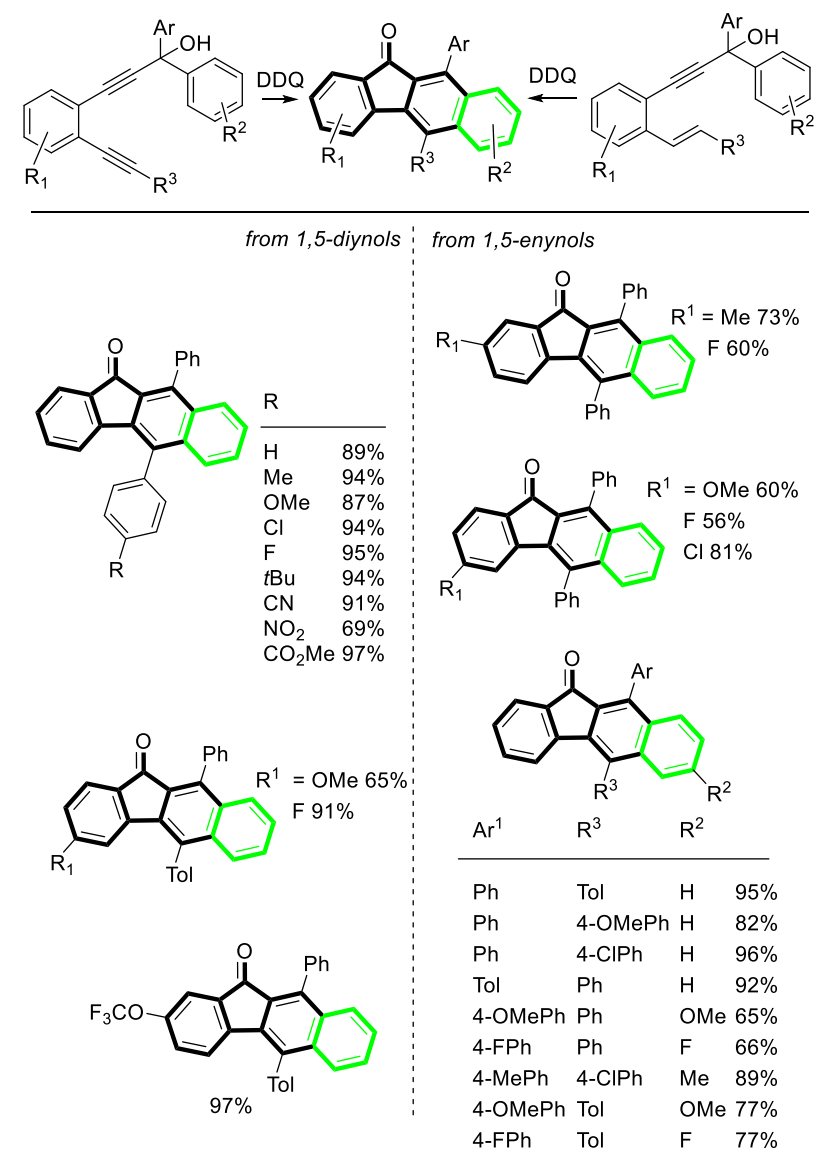

SCHEME 34 - Cycloaromatization of 1,5-enynols and 1,5-diynols

Finally, naphtho[b]fluorenones have been prepared using a $[2+2+2]$ cycloaddition reaction from benzocyclopentynone surrogate as a $2 \pi$ partner (Scheme 35 ). The cycloaddition of the selected diyne afforded the partially hydrogenated pentacyclic architecture. The latter undergoes aromatization using DDQ to yield the naphtho[b]fluorenone in $37 \%$ yield ( 2 steps). ${ }^{75}$ 


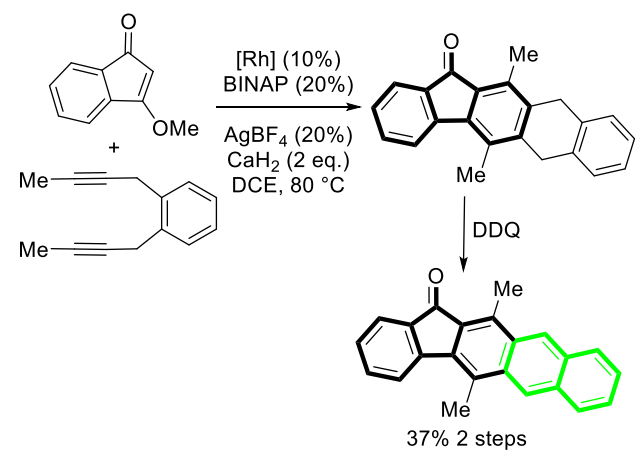

SCHEME 35 - Naphtho[b]fluorenone using $[2+2+2]$ cycloaddition reaction from benzocyclopentynone surrogate

\section{Conclusion}

The attractive properties of extended fluorenones have called for the development of new, efficient and straightforward methodologies for the construction of such molecular platforms. The synthesis of "regular" tricyclic fluorenones from benzene precursors is a well-established approach, however, the formation of extended fluorenones often requires a naphthalene derivative (or a higher homologue of benzene) as a starting material. The fact that the experimental conditions are not easily transferrable from benzene to higher aromatics represents a major obstacle to the development of extended fluorenones, the latter often being overlooked when designing the synthetic methodologies. In this context, the synthesis of extended fluorenones often appears as only "complementary" to the synthesis of "regular" ones, despites having, as an example, enhanced electronic properties due to their larger aromatic system.

Nevertheless, various approaches towards this family of compounds have been reported over the years, leading to a wide molecular diversity. Current strategies now allow for the synthesis of more intricate, tetra- and pentacyclic fluorenones. These strategies address the construction of the main skeleton by creating the central rings of each of target, sometimes jointly, in a very efficient and selective way, which give rise to extended fluorenones bearing multiple functions and substituents. But there is still a lot to discover about extended fluorenones and their synthesis and it is expected that even more innovative, straightforward, and environment-friendly synthetic strategies will emerge, as we will most certainly need the numerous appealing properties of extended fluorenones. As examples, research dedicated to the selective installation of various substituents on aromatic polycycles is likely to increase the molecular diversity of extended fluorenones, to bring electronic and steric modulations to the central fluorenone fragment or on the peripheral rings. Two axes seem unavoidable, involving the development of tools and synthesis strategies either to diversify the pool of polycyclic precursors before the assembly of the fluorenone core, or the late functionalization of pre-existing extended fluorenones. Among the former, the emergence of one-pot strategies for the construction of one or more cycles of an extended fluorenone undoubtedly constitutes an interesting avenue of reflection to expand the number, the size and diversity of extended fluorenones. 


\section{Acknowledgments}

The authors would like to thank Natalia Baranska for taking the time to proofread this manuscript.

\section{Keywords}

Arenes - Annulation - Aromaticity - Carbocycles - Cyclization - Fluorenones

\footnotetext{
${ }^{1}$ R. Fittig, Berichte der Dtsch. Chem. Gesellschaft 1873, 6, 187-187.

2 T.-T. Do, J. Subbiah, S. Manzhos, D. J. Jones, J. M. Bell, P. Sonar, Org. Electron. 2018, 62, 12-20.
}

${ }^{3}$ a) X. Chen, K.-M. Engle, D.-H. Wang, J.-Q. Yu, Angew. Chem. Int. Ed. 2009, 48, 5094-5115; b) J. Wencel Delord, T. Dröge, F. Glorius, Chem. Soc. Rev. 2011, 40, 4740-4761; c) D. Y.-K. Chen, S. W. Youn, Chem. Eur. J. 2012, 18, 9452-9474; d) J. Yamaguchi, A. D. Yamaguchi, K. Itami, Angew. Chem. Int. Ed. 2012, 51, 8960-9009; e)T. Brückl, R. D. Baxter, Y. Ishihara, P. S. Baran, Acc. Chem. Res. 2012, 45, 826-839.

${ }^{4}$ H. Su, R. Liu, M. Shu, M. Tang, J. Wang, H. Zhu, Dye. Pigment. 2019, 162, 52-58.

${ }^{5}$ R. Demadrille, B. Divisia-Blohorn, M. Zagorska, S. Quillard, S. Lefrant, A. Pron, Electrochimica acta 2005, 50, 1597-1603.

${ }^{6}$ C. Wang, A. S. Batsanov, M. R. Bryce, I. Sage, Org. Lett. 2004, 6, 2181-2184.

${ }^{7}$ I.-S. Tamgho, S. Chaudhuri, M. Verderame, D. J. DiScenza, M. Levine, RSC Adv. 2017, 7, 28489-28493.

${ }^{8}$ a) T. Link, L. E. Overman, in Metal-catalyzed Cross-coupling Reactions, (Eds: F. Diederich, P. J. Stang), WileyVCH, Weinheim, Germany 2007, Ch. 6, 230-269. b) Z.-P. Li, D.-S. Bohle, C.-J. Li, Proc. Natl. Acad. Sci. U.S.A. 2006, 103, 8928-8933.

${ }^{9}$ a) C. Fan, W. Wang, Y. Wang, G. Qin, W. Zhao, Phytochemistry 2001, 57, 1255-1258; b) T. Akiyama, K. T. Nakamura, Y. Takahashi, H. Naganawa, Y. Muraoka, T. Aoyagi, T. Takeuchi, J. Antibiot 1998, 51, 586-588; c) X. Y. Wu, G. W. Qin, D. J. Fan, R. S. Xu, Phytochemistry 1994, 36, 477-479; d) M. C. Cone, C. R. Melville, M. P. Gore, S. J. Gould, J. Org. Chem. 1993, 58, 1058-1061; e) M. V. J. Sargent, Chem. Soc., Perkin Trans. 1 1987, 2553-2563; f) S. K. Talapatra, S. Bose, A. K. Mallik, B. Talapatra, Tetrahedron 1985, 41, 2765-2769.

${ }^{10}$ For recent review on the fluorenone see S. Patel, B. Rathod, S. Regu, S. Chak, A. Shard, ChemistrySelect 2020, 5, 10673-10691.

${ }^{11}$ X. Pang, Y. Tan, C. Tan, W. Li, N. Du, Y. Lu, Y. Jiang, ACS App. Mater. Interfaces 2019, 11, 28246-28253.

${ }^{12}$ E. H. Huntress, E. B. Hershberg, I. S. Cliff, J. Am. Chem. Soc. 1931, 53, 2720-2724.

${ }^{13}$ L. C. Finney, L. J. Mitchell, C. J. Moody, Green Chem. 2018, 20, 2242-2249.

${ }^{14}$ S. Nakai, T. Uematsu, Y. Ogasawara, K. Suzuki, K. Yamaguchi, N. Mizuno, ChemCatChem 2018, 10,1096 1106

${ }^{15}$ a) X. Zhang, X. Ji, R. Su, B. L. Weeks, Z. Zhang, S. Deng, ChemPlusChem 2013, 78, 703-711; b) X. Zhang, X. Ji, S. Jiang, L. Liu, B. L. Weeks, Z. Zhang, GreenChem. 2011, 13, 1891-1896.

${ }^{16}$ K. Yamadaa, H. Shibamotoa, Y. Tanigawaa, H. Ishikawaa, J.-I. Nishidaa, C. Kitamurab, H. Kuratac, T. Kawase, Synlett 2016, 27, 2085-2090.

${ }^{17}$ B. K. Banik, M. S. Venkatraman, C. Mukhopadhyay, F. F. Becker, Tetrahedron Lett. 1998, 39, 7247-7250.

${ }^{18}$ Y. Tian, K. Uchida, H. Kurata, Y. Hirao, T. Nishiuchi, T. Kubo, J. Am. Chem. Soc. 2014, 136, 12784-12793.

${ }^{19}$ A. Patra, S. Ghorai, S. De, D. Mal, Synthesis 2006, 2006, 2556-2562.

${ }^{20}$ P. Gandeepan, C.-F. Hung, C.-H. Cheng, Chem. Commun. 2012, 48, 9379-9381.

${ }^{21}$ H. Li, R.-Y. Zhu, W.-J. Shi, K.-H. He, Z.-J. Shi, Org Lett. 2012, 14, 4850-4853.

${ }^{22}$ R. Kishore, S. S. Priya, M. Sudhakar, B. Venu, A. Venugopal, J. Yadavb, M. Lakshmi Kantam, Catal. Sci. Technol. 2015, 5, 3363-3367.

${ }^{23}$ B. Large, N. Gigant, D. Joseph, G. Clavier, D. Prim, Eur. J. Org. Chem. 2019, 1835-1841.

${ }^{24}$ B. Large, N. Gigant, D. Joseph, G. Clavier, D. Prim, Eur. J. Org. Chem. 2019, 6407-6412.

${ }^{25}$ A. Vignesh, W. Kaminsky, N. Dharmaraj, ChemCatChem 2016, 8, 3207-3212.

${ }^{26}$ A. Patel, M. Shaikh, K. Chikhalia, Tetrahedron 2019, 75, 236-245. 
${ }^{27}$ Q. Gao, S. Xu, Org. Biomol. Chem. 2018, 16, 208-212.

${ }^{28}$ S. Seo, M. Slater, M. F. Greaney, Org. Lett. 2012, 14, 2650-2653.

${ }^{29}$ Z. Shi, F. Glorius, Chem. Sci. 2013, 4, 829-833.

${ }^{30}$ T.-P. Liu, Y.-X. Liao, C.-H. Xing, Q.-S. Hu, Org Lett. 2011, 13, 2452-2455.

${ }^{31}$ Y.-B. Zhao, B. Mariampillai, D. A. Candito, B. Laleu, M. Li, M. Lautens, Angew. Chem. Int. Ed. 2009, 48, 18491852.

${ }^{32}$ S. Sarkar, N. Tadigoppula, RSC Adv. 2014, 4, 40964-40968.

33 J. Zhao, D. Yue, M. A. Campo, R. C. Larock, J. Am. Chem. Soc. 2007, 129, 5288-5295.

${ }^{34}$ H. Konishi, S. Futamata, X. Wang, K. Manabe, Adv. Synth. Catal. 2018, 360, 1805-1809.

${ }^{35}$ V. S. Thirunavukkarasu, C.-H. Cheng, Chem. Eur. J. 2011, 17, 14723-14726.

${ }^{36}$ V. S. Thirunavukkarasu, K. Parthasarathy, C.-H. Cheng, Angew. Chem. Int. Ed. 2008, 47, 9462-9465.

${ }^{37}$ T. Fukuyama, S. Maetani, K. Miyagawa, I Ryu, Org. Lett. 2014, 16, 3216-3219.

${ }^{38}$ R. Ruzi, M. Zhang, K. Ablajan, C. Zhu, J. Org. Chem. 2017, 82, 12834-12839.

${ }^{39}$ D. Sun, B. Li, J. Lan, Q. Huang, J. You, Chem. Commun. 2016, 52, 3635-3638.

${ }^{40}$ L. Tejerina, M. Victoria Martínez-Díaz, T. Torres, Org. Lett. 2019, 21, 8, 2908-2912

${ }^{41}$ E. Bamberger, C. Burgdorf, Chem. Ber. 1890, 23, 2433-2446.

${ }^{42}$ a) C. Graebe, Chem. Ber. 1900, 33, 680-681 ; b) K. Ko-Hoon, K. Sung-Hwan, L. Ka-Young, K. Jae-Nyoung, Bull. Korean Chem. Soc. 2011, 32, 1387-1390 ; c) J. E. Barker, C. K. Frederickson, M. H. Jones, L. N. Zakharov, M. M. Haley, Org. Lett. 2017, 19, 5312-5315 ; d) H. Miyoshi, M. Miki, S. Hirano, A. Shimizu, R. Kishi, K. Fukuda, D. Shiomi, K. Sato, T. Takui, I. Hisaki, M. Nakano, Y. Tobe, J. Org. Chem. 2017, 82, 1380-1388; e) A. S. Hacker, M. Pavano, J. E. Wood, C. E. Immoos, H. Hashimoto, S. P. Genis, D. K. Frantz, J. Org. Chem. 2018, 83, 510-515. ${ }^{43}$ L. Chardonnens, H. Chardonnens, Helv. Chim. Acta 1958, 41, 1606-1626.

${ }^{44}$ a) W. Carruthers, R. Pooranamoorthy, J. Chem. Soc., Perkin transactions / 1974, 2405-2409 ; b) C. I. Martins, P. J. Coelho, L. M. Carvalho, A. M.F. Oliveira-Campos, A. Samat, R. Guglielmetti, Helv. Chim. Acta 2003, 86, 570578; c) C. K. Frederickson, L. N. Zakharov, M. M. Haley, J. Am. Chem. Soc. 2016, 138, 16827-16838.

${ }^{45}$ A. Streitwieser, S. M. Brown, J. Org. Chem. 1988, 53, 904-906.

${ }^{46}$ S. J. Gould, J. Chen, M. C. Cone, M. P. Gore, C. R. Melville, N. Tamayo, J. Org. Chem. 1996, 61, 5720-5721.

${ }^{47}$ O. Khdour, E. B. Skibo, Org. Biomol. Chem. 2009, 7, 2140-2154.

${ }^{48}$ K. Luo, T. Cao, H. Jiang, L. Chen, S. Zhu, Org. Lett. 2017, 19, 5856-5859.

49 a) T. Jousselin-Oba, M. Mamada, A. Okazawa, J. Marrot, T. Ishida, C. Adachi, A. Yassar, M. Frigoli, Chem. Sci. 2020, 11, 12194-12205 ; b) T. Jousselin-Oba, P. E. Deal, A. G. Fix, C. K. Frederickson, C. L. Vonnegut, A. Yassar, L. N. Zakharov, M. Frigoli, M. M. Haley, Chem. Asian J. 2019, 14, 1737-1744 ; c) K. Sbargoud, M. Mamada, J. Marrot, S. Tokito, A. Yassar, M. Frigoli, Chem. Sci. 2015, 6, 3402-3409.

${ }^{50}$ M. Guerola, M. Sánchez-Rosello, C. Mulet, C. del Pozo, S. Fustero, Org. Lett. 2015, 17, 960-963.

${ }^{51}$ F. Péron, C. Fossey, T. Cailly, F. Fabis, Org. Lett. 2012, 14, 1827- 1829.

52 Y.-B. Zhao, B. Mariampillai, D. A. Candito, B. Laleu, M. Li, M. Lautens, Angew. Chem. Int. Ed. 2009, 48, 18491852.

${ }^{53}$ Y. Tian, K. Uchida, H. Kurata, Y. Hirao, T. Nishiuchi, T. Kubo, J. Am. Chem. Soc. 2014, 136, 12784-12793.

${ }^{54}$ S. Qiu, S. Zhai, H. Wang, X. Chen, H. Zhai, Chem. Commun. 2019, 55, 4206-4209.

${ }^{55}$ H.-C. Wan, J.-M. Huang, Y.-H. Jhan, J.-C. Hsieh, Org. Lett. 2013, 15, 2742-2745.

${ }^{56}$ S. Sarkar, M. Jana, T. Narender, Eur. J. Org. Chem. 2013, 6491-6495.

${ }^{57}$ K. H. Kim, S. H. Kim, K Y. Lee, J. N. Kim, Bull. Korean Chem. Soc. 2011, 32, 1387-1390

58 J. Wang, J. Xiang, M. Wang, J. Guan, A. Wu, Tetrahedron 2014, 70, 1412-1417.

${ }^{59}$ L. Liu, J. Qiang, S. Bai, Y. Li, C. Miao, J. Li, Appl Organometal Chem. 2017, 31, e3817.

${ }^{60}$ L. Liu, J. Qiang, S. Bai, Y. Li, C. Miao, J. Li, Appl Organometal Chem. 2017, 31, e3817.

61 J. P. Waldo, X. Zhang, F. Shi, R. C. Larock, J. Org. Chem. 2008, 73, 6679-6685.

${ }^{62}$ X. Zhang, R. C. Larock, Org Lett. 2005, 7, 3973-3976.

63 J. L. Morris, C. L. Becker, F. R. Fronczek, W. H. Daly, M. L. McLaughlin, J. Org. Chem. 1994, 59, 6484-6486.

${ }^{64}$ R. L. Danheiser, A. E. Gould, R. F. de la Pradilla, A. L. Helgason, J. Org. Chem. 1994, 59, 5514-5515.

${ }^{65}$ W.-J. Hao, P. Zhou, Z. Yang, J.-P. Zhang, S.-J. Tu, Tetrahedron 2016, 71, 2206-2212.

${ }^{66}$ J. Zhang, H. Wang, S. Ren, W. Zhang, Y. Liu, Org. Lett. 2015, 17, 2920-2923.

${ }^{67}$ J. Zhang, H. Zhang, D. Shi, H. Jin, Y. Liu, Eur. J. Org. Chem. 2016, 5545-5558.

${ }^{68}$ E. González-Cantalapiedra, Ó. de Frutos, C. Atienza, C. Mateo, A. M. Echavarren, Eur. J. Org. Chem. 2006, 1430-1443.

${ }^{69}$ C. Atienza, C. Mateo, Ó. de Frutos, A. M. Echavarren, Org. Lett. 2001, 3, 153-155.

${ }^{70}$ D. Rodríguez, M. F. Martínez-Esperón, A. Navarro-Vázquez, L. Castedo, D. Domínguez, C. Saá, J. Org. Chem. 2004, 69, 3842- 3848. 
71 J. Tan, Z. Wang, J. Yuan, Y. Peng, Z. Chen, Adv. Synth. Catal. 2019, 361, 1295-1300.

72 M. Mandal, S. Sakthivel, R. Balamurugan, J. Org. Chem. 2021, 86, 333-351.

${ }^{73}$ B. Yan, Y. Fu, H. Zhu, Z. Chen, J. Org. Chem. 2019, 84, 4246-4262.

${ }^{74}$ H. Zhu, Z. Chen, Org. Lett. 2016, 18, 488-491.

${ }^{75}$ A.-D. Manick, B. Salgues, J.-L. Parrain, E. Zaborova, F. Fages, M. Amatore, L. Commeiras, Org. Lett. 2020, 22, 1894-1898. 